COMMUNICATIONS IN

ANALYSIS AND GEOMETRY

Volume 14, Number 3, 443-474, 2006

\title{
Covariant Poisson Structures on Complex Grassmannians
}

\author{
N. CiCCOli $^{1}$ AND A. J.-L. SheU ${ }^{2}$
}

The purpose of this paper is to study covariant Poisson structures on $G r_{k}^{n} \mathbb{C}$ obtained as quotients by coisotropic subgroups of the standard Poisson-Lie $S U(n)$. Properties of Poisson quotients allow to describe Poisson embeddings generalizing those obtained in [20].

\section{Introduction.}

In [20] a family of covariant Poisson structures on complex projective spaces underlying the Dijkhuizen-Noumi quantization [6] was studied from the point of view of coisotropic subgroups with respect to an affine Poisson structure on $S U(n)$, providing also a description of the associated Lagrangian subalgebras and Poisson embeddings of standard odd Poisson spheres in non standard Poisson projective spaces.

In this paper, we plan to extend those results to complex Grassmannians (their quantum version may be found in [7]). The emphasis is laid even more strongly on the role played by subgroups which are coisotropic with respect to the standard multiplicative Poisson structure on $S U(n)$.

One reason of interest lies in the fact that every coisotropic subgroup of a Poisson-Lie group can be quantized in such a way as to fit in a nice duality diagram [4]. Furthermore, coisotropic submanifolds have recently raised a lot of attention in the context of deformation quantization [3, 2] and played a role in the analysis of Poisson sigma-models over group manifolds [1].

In the first section we clarify the relation between coisotropic subgroups of a Poisson-Lie group and coisotropic subgroups of translated affine Poisson bivectors. This relates results in [20] with those in the present work, allowing a natural interpretation from the foliation point of view.

\footnotetext{
${ }^{1}$ supported by PRIN Azioni di gruppi su varietá and GNSAGA.

${ }^{2}$ supported by the University of Kansas General Research Fund allocation \#2301 for FY 2004.
} 
In the second section, we describe the family of covariant Poisson structures on complex Grassmannians under consideration and show how it can be obtained as quotients by coisotropic subgroups. Such structure was first introduced in [12] under different methods. A specific non standard Grassmannian was studied, recently, with Lie group methods by Foth and $\mathrm{Lu}$ (see $[10])$.

Finally, in the last section, we describe a general procedure allowing to determine Poisson embeddings of $G$-spaces. Applying it to projective spaces, we show how it recovers the whole symplectic foliation in the standard case and the Poisson embeddings of [20] in the non-standard one. Moving on to Grassmannians such procedure will give embeddings of standard PoissonStiefel manifolds (and of other more general manifolds) in non-standard Poisson Grassmannians. In the special case of Grassmannians $G_{m}^{2 m}(\mathbb{C})$ this will result in a Poisson embedding of the standard Poisson-Lie group $U(m)$. Such embeddings are relevant also from the point of view of quantum spaces, where they were first identified. It is noted in the study of the quantum algebra $\mathcal{C}\left(\mathbb{P}_{q, c}^{n}\right)$ in the context of groupoid $C^{*}$-algebras carried out in $[18,19]$, where they lead to the construction of some composition sequences for the algebra. We expect that quantum Stiefel manifolds studied in [17] and suitable generalizations will appear as quotients of nonstandard complex $q$-Grassmannians and allow a similar detailed analysis.

\section{Coisotropic and Affine Coisotropic Subgroups.}

\subsection{Affine Poisson Structures.}

Let $G$ be a given Lie group, with Lie algebra $\mathfrak{g}$. In the following, we use $R_{g}$ (resp. $L_{g}$ ) to denote the right (resp. left) translation action on $G$ by $g \in G$, and also all the actions induced by it on tensors of $G$, e.g. $R_{g}(v)=\left(D\left(R_{g}\right)\right)_{x}(v) \in T_{x g} G$ for any vector $v \in T_{x} G$, and $\left(R_{g} X\right)(x)=$ $\left(D\left(R_{g}\right)\right)_{x g^{-1}}\left(X\left(x g^{-1}\right)\right) \in T_{x} G$ for any vector field $X \in \Gamma(T G)$, where $D\left(R_{g}\right)$ is the differential (a vector bundle map on $T G$ ) of the diffeomorphism $R_{g}$. Note that the right translation $R$ is an anti-homomorphism, i.e. $R_{g} R_{h}=R_{h g}$. Similarly, we have the left translation $L_{x}$, but $L$ is a homomorphism, i.e. $L_{g} L_{h}=L_{g h}$. Given any 2-tensor $\rho: G \rightarrow \wedge^{2} T G$ let $\tilde{\rho}(g):=L_{g}^{-1} \rho(g) \in \wedge^{2} \mathfrak{g}$.

First, we recall the following facts for an (alternating) 2-tensor field $\rho$ on a Lie group $G$ (see $[13,22]$ ). 
(1) $\rho$ is called multiplicative if

$$
\rho(g h)=L_{g}(\rho(h))+R_{h}(\rho(g)) .
$$

(Note that $\rho(e)=0$ if $\rho$ is multiplicative, where $e$ is the unit of $G$.)

(2) $\rho$ is called affine if

$$
\rho(g h)=L_{g}(\rho(h))+R_{h}(\rho(g))-L_{g} R_{h}(\rho(e)) .
$$

(3) $\rho$ is affine if and only if $\pi:=\rho-(\rho(e))^{l}$ is multiplicative, where $X^{l}$ denotes the left-invariant tensor field generated by $X \in \wedge^{2} \mathfrak{g}$. (Note that for any 2-tensor field $\pi$ with $\pi(e)=0$ and $X \in \wedge^{2} \mathfrak{g}$, if $\rho:=\pi+X^{l}$, then $\rho(e)=X$ and hence $\pi=\rho-(\rho(e))^{l}$. So all affine $\rho$ are of the form $\rho=\pi+X^{l}$ for some multiplicative $\pi$ and $X \in \wedge^{2} \mathfrak{g}$.)

(4) If $\pi$ is a Poisson-Lie structure on $G$, then $\rho=\pi+X^{l}$ (with $\rho(e)=X$ ) is affine for any $X \in \wedge^{2} \mathfrak{g}$ (but may not be Poisson); in this case, $\rho$ is also Poisson if and only if $d X=\frac{1}{2}[X, X]([5])$.

(5) If $\rho$ is affine Poisson, then $\pi:=\rho-(\rho(e))^{l}$ is multiplicative and Poisson. (But the converse may not be true, cf. (4) above.)

(6) Given $\rho$ Poisson, we have that $\rho$ is affine Poisson if and only if $\pi:=\rho-(\rho(e))^{l}$ is multiplicative Poisson (or Poisson-Lie).

Now, we show that if $\rho$ is affine Poisson and $\rho(\sigma)=0$ for some point $\sigma \in G$, then $R_{\sigma^{-1}} \rho$ is Poisson-Lie.

Lemma 2.1. If $\rho$ is affine Poisson, then $R_{\sigma} \rho$ is also affine Poisson for any $\sigma \in G$. (We don't assume $\rho(\sigma)=0$ in this lemma.)

Proof. Clearly, the right translation of a Poisson structure on $G$ is still a Poisson structure. So $R_{\sigma} \rho$ is Poisson. Now, by the commutativity $R_{g} L_{h}=$ $L_{h} R_{g}$ for all $g, h \in G$, we get

$$
\begin{gathered}
{\left[R_{\sigma} \rho-\left(\left(R_{\sigma} \rho\right)(e)\right)^{l}\right](g)=R_{\sigma}\left(\rho\left(g \sigma^{-1}\right)\right)-L_{g}\left(\left(R_{\sigma} \rho\right)(e)\right)} \\
=R_{\sigma}\left[L_{g}\left(\rho\left(\sigma^{-1}\right)\right)+R_{\sigma^{-1}}(\rho(g))-L_{g} R_{\sigma^{-1}}(\rho(e))\right]-L_{g}\left(R_{\sigma}\left(\rho\left(\sigma^{-1}\right)\right)\right) \\
=R_{\sigma}\left(L_{g}\left(\rho\left(\sigma^{-1}\right)\right)\right)+\rho(g)-L_{g}(\rho(e))-L_{g}\left(R_{\sigma}\left(\rho\left(\sigma^{-1}\right)\right)\right) \\
=\rho(g)-L_{g}(\rho(e))=\left[\rho-(\rho(e))^{l}\right](g)
\end{gathered}
$$


which shows that

$$
R_{\sigma} \rho-\left(\left(R_{\sigma} \rho\right)(e)\right)^{l}=\rho-(\rho(e))^{l}
$$

a multiplicative Poisson structure since $\rho$ is affine Poisson. Thus, $R_{\sigma} \rho$ is affine Poisson.

Proposition 2.2. If $\rho$ is affine Poisson and $\rho(\sigma)=0$ for some point $\sigma \in G$, then $R_{\sigma^{-1}} \rho$ is Poisson-Lie.

Proof. $R_{\sigma^{-1}} \rho$ is affine Poisson with

$$
\left(R_{\sigma^{-1}} \rho\right)(e)=R_{\sigma^{-1}}(\rho(\sigma))=0
$$

and hence $R_{\sigma^{-1}} \rho$ is multiplicative Poisson.

\subsection{Coisotropic Subgroups.}

In this section, we will clarify the relation between affine Poisson structures on Lie groups and coisotropic subgroups of Poisson-Lie groups, introducing the notion of affinely coisotropic subgroup.

Recall that for a given Poisson manifold $\left(M, \pi_{M}\right)$ a coisotropic submanifold is an embedded submanifold such that its defining ideal (i.e. the ideal of smooth functions which are zero on the manifold) is a Poisson subalgebra. For a given Poisson-Lie group $(G, \pi)$ a coisotropic subgroup is a Lie subgroup $H$ which is also a coisotropic submanifold. At the infinitesimal level, if $\delta=(D \tilde{\pi})_{e}: \mathfrak{g} \rightarrow \wedge^{2} \mathfrak{g}$ represents the cobracket and $\mathfrak{h}$ is a Lie subalgebra of $\mathfrak{g}$ then $\mathfrak{h}$ can be integrated to a coisotropic subgroup if and only if $\delta(\mathfrak{h}) \subseteq \mathfrak{h} \wedge \mathfrak{g}$

Let $\rho$ be an affine Poisson structure on the Lie group $G$ and let $H$ be a closed (connected) subgroup. It is known that the multiplicative Poisson structure on $G$ induces (or projects to) a well-defined Poisson structure on $G / H$ when $H$ is a coisotropic subgroup.

The concept of a coisotropic subgroup $H$ of an affine Poisson Lie group $(G, \rho)$ is more delicate, and there is a fine distinction between "a coisotropic subgroup" and "a subgroup that is a coisotropic submanifold" as discussed below. First, we note that the following conditions are equivalent:

(2) $\rho(h)-R_{h}(\rho(e)) \in L_{h}(\mathfrak{h} \wedge \mathfrak{g})$ for all $h \in H$;

(3) $\rho(k h)-R_{h}(\rho(k)) \in L_{k h}(\mathfrak{h} \wedge \mathfrak{g})$ for all $h, k \in H$; 
(4) $\left((D \tilde{\rho})_{e}+[\tilde{\rho}(e), \cdot]\right)(\mathfrak{h}) \subset \mathfrak{h} \wedge \mathfrak{g}$

(5) $\rho(g h)-R_{h}(\rho(g)) \in L_{g h}(\mathfrak{h} \wedge \mathfrak{g})$ for all $h \in H$ and $g \in G$.

Furthermore, if $\operatorname{ad}_{\mathfrak{h}}(\rho(e)) \subseteq \mathfrak{h} \wedge \mathfrak{g}$ such conditions are equivalent to

(1) $H$ is a $\rho$-coisotropic submanifold of $G$, i.e. $\rho(h) \in L_{h}(\mathfrak{h} \wedge \mathfrak{g})$ for all $h \in H$;

In fact,

$$
R_{h}(\rho(e))=L_{h}\left(L_{h}^{-1} R_{h}(\rho(e))\right)=L_{h}\left(\operatorname{Ad}_{h}^{-1}(\rho(e))\right)
$$

and so $(1) \Leftrightarrow(2)$ if $\operatorname{ad}_{\mathfrak{h}}(\rho(e)) \subset \mathfrak{h} \wedge \mathfrak{g}$. Since $\rho$ is affine, we have

$$
L_{g}\left[\rho(h)-R_{h}(\rho(e))\right]=\rho(g h)-R_{h}(\rho(g))
$$

for any $h \in H$ and $g \in G$, and hence (2), (3), and (5) are clearly equivalent. From

$$
L_{h}^{-1}\left[\rho(h)-R_{h}(\rho(e))\right]=L_{h^{-1}} \rho(h)-\operatorname{Ad}_{h}^{-1}(\rho(e))
$$

and $D\left[\operatorname{Ad}_{h}^{-1}(\rho(e))\right]_{h=e}=-\operatorname{ad} .(\rho(e))$, it is not hard to see the equivalence of (3) and (4).

Note that even if $\rho$ is multiplicative and a subgroup $H$ is a $\rho$-coisotropic submanifold, a coset $g H$ of $H$ in general need not be a $\rho$-coisotropic submanifold of $G$, but is "affinely (or relatively) $\rho$-coisotropic" in the sense of condition (5). Note that in general, when $\rho(e) \neq 0$, i.e. $\rho$ is not multiplicative, both $(1) \Rightarrow(2)$ and $(2) \Rightarrow(1)$ may not hold. We define a closed subgroup $H$ of an (affine) Poisson Lie group $G$ to be a $\rho$-coisotropic subgroup if each coset $g H$ with $g \in G$ is an affinely $\rho$-coisotropic submanifold of $G$, i.e. $\rho(g h)-R_{h}(\rho(g)) \in L_{g h}(\mathfrak{h} \wedge \mathfrak{g})$ for all $h \in H$. So, when $\rho$ is multiplicative, a closed subgroup $H$ of $G$ is a $\rho$-coisotropic submanifold of $G$ if and only if $H$ is a $\rho$-coisotropic subgroup of $G$.

Theorem 2.3. Let $\pi$ be a Poisson 2-tensor on a Lie group G. For a closed Lie subgroup $H$ of $G$ and $\sigma \in G$, the conjugate $\operatorname{Ad}_{\sigma} H$ of $H$ is $\pi$-coisotropic if and only if $H$ is $\pi_{\sigma}$-coisotropic, where $\pi_{\sigma}(g):=R_{\sigma}\left(\pi\left(g \sigma^{-1}\right)\right)$ for $g \in G$.

Proof. Let $\tilde{\pi}(g):=L_{g}^{-1}(\pi(g)) \in \wedge^{2} \mathfrak{g}$. Since

$$
\begin{aligned}
L_{g h}^{-1}\left[\pi(g h)-R_{h} \pi(g)\right] & =\tilde{\pi}(g h)-L_{h}^{-1} L_{g}^{-1} R_{h} \pi(g) \\
& =\tilde{\pi}(g h)-L_{h}^{-1} R_{h} L_{g}^{-1} \pi(g) \\
& =\tilde{\pi}(g h)-L_{h}^{-1} R_{h} \tilde{\pi}(g)=\tilde{\pi}(g h)-\operatorname{Ad}_{h}^{-1}(\tilde{\pi}(g)),
\end{aligned}
$$


a subgroup $H$ is $\pi$-coisotropic if and only if

$$
\tilde{\pi}(g h)-\operatorname{Ad}_{h}^{-1}(\tilde{\pi}(g)) \in \mathfrak{h} \wedge \mathfrak{g}
$$

for all $g \in G$ and $h \in H$. Thus, $\operatorname{Ad}_{\sigma} H$ is $\pi$-coisotropic if and only if

$$
(*) \tilde{\pi}\left(g \operatorname{Ad}_{\sigma}(h)\right)-\operatorname{Ad}_{\operatorname{Ad}_{\sigma}(h)}^{-1}(\tilde{\pi}(g)) \in \operatorname{Ad}_{\sigma}(\mathfrak{h}) \wedge \mathfrak{g}
$$

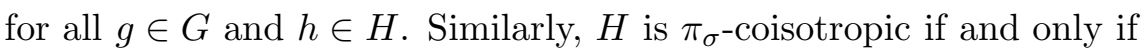

$$
(* *) \widetilde{\pi_{\sigma}}(g h)-\operatorname{Ad}_{h}^{-1}\left(\widetilde{\pi_{\sigma}}(g)\right) \in \mathfrak{h} \wedge \mathfrak{g}
$$

for all $g \in G$ and $h \in H$. Note that

$$
\begin{aligned}
\widetilde{\pi_{\sigma}}(g) & =L_{g}^{-1}\left(\pi_{\sigma}(g)\right)=L_{g}^{-1}\left(R_{\sigma} \pi\left(g \sigma^{-1}\right)\right)=R_{\sigma} L_{g}^{-1} \pi\left(g \sigma^{-1}\right) \\
& =R_{\sigma} L_{\sigma}^{-1} L_{\sigma} L_{g}^{-1} \pi\left(g \sigma^{-1}\right)=R_{\sigma} L_{\sigma}^{-1} L_{g \sigma^{-1}}^{-1} \pi\left(g \sigma^{-1}\right) \\
& =R_{\sigma} L_{\sigma}^{-1} \tilde{\pi}\left(g \sigma^{-1}\right)=\operatorname{Ad}_{\sigma}^{-1}\left(\tilde{\pi}\left(g \sigma^{-1}\right)\right)
\end{aligned}
$$

for any $g \in G$. So

$$
\begin{gathered}
\widetilde{\pi_{\sigma}}(g h)-\operatorname{Ad}_{h}^{-1}\left(\widetilde{\pi_{\sigma}}(g)\right)=\operatorname{Ad}_{\sigma}^{-1}\left(\tilde{\pi}\left(g h \sigma^{-1}\right)\right) \\
\quad-\operatorname{Ad}_{h}^{-1}\left(\operatorname{Ad}_{\sigma}^{-1}\left(\tilde{\pi}\left(g \sigma^{-1}\right)\right)\right) \\
=\operatorname{Ad}_{\sigma}^{-1}\left(\tilde{\pi}\left(g \sigma^{-1} \operatorname{Ad}_{\sigma}(h)\right)\right) \\
\quad-\operatorname{Ad}_{\sigma}^{-1} \operatorname{Ad}_{\sigma} \operatorname{Ad}_{h}^{-1} \operatorname{Ad}_{\sigma}^{-1}\left(\tilde{\pi}\left(g \sigma^{-1}\right)\right) \\
=\operatorname{Ad}_{\sigma}^{-1}\left[\left(\tilde{\pi}\left(g \sigma^{-1} \operatorname{Ad}_{\sigma}(h)\right)\right)\right. \\
\left.-\operatorname{Ad}_{\sigma h \sigma^{-1}}^{-1}\left(\tilde{\pi}\left(g \sigma^{-1}\right)\right)\right]
\end{gathered}
$$

and hence the condition $(* *)$ is equivalent to

$$
\tilde{\pi}\left(g \sigma^{-1} \operatorname{Ad}_{\sigma}(h)\right)-\operatorname{Ad}_{\operatorname{Ad}_{\sigma} h}^{-1}\left(\tilde{\pi}\left(g \sigma^{-1}\right)\right) \in \operatorname{Ad}_{\sigma}(\mathfrak{h} \wedge \mathfrak{g})=\operatorname{Ad}_{\sigma}(\mathfrak{h}) \wedge \mathfrak{g}
$$

for all $g \in G$ and $h \in H$, or equivalently, the condition $\left(^{*}\right)$.

Proposition 2.4. Let $G$ be a Poisson-Lie group, $H$ a closed subgroup such that its conjugate $H_{\sigma}:=\operatorname{Ad}_{\sigma} H=\sigma H \sigma^{-1}$ is coisotropic where $\sigma \in G$. Let $\pi_{\sigma}$ be the affine Poisson structure on $G$ given by $\pi_{\sigma}(g):=R_{\sigma} \pi\left(g \sigma^{-1}\right)$. Let $p: G \rightarrow G / H$ and $p_{\sigma}: G \rightarrow G / H_{\sigma}$ be the natural projections. Then, the Poisson manifolds $\left(G / H, p_{*} \pi_{\sigma}\right)$ and $\left(G / H_{\sigma},\left(p_{\sigma}\right)_{*} \pi\right)$ are Poisson diffeomorphic. 
Proof. There is a natural diffeomorphism between $G / H$ and $G / H_{\sigma}$ given by $\iota:[g]_{H} \mapsto\left[g \sigma^{-1}\right]_{H_{\sigma}}$ which satisfies

$$
p_{\sigma}=\iota \circ p \circ R_{\sigma}
$$

where $[g]_{H}:=g H \in G / H$. We claim that $\iota$ is a Poisson map, i.e. $\iota_{*}\left(p_{*} \pi_{\sigma}\right)=\left(p_{\sigma}\right)_{*} \pi$. Indeed,

$$
\begin{aligned}
\iota_{*}\left(p_{*} \pi_{\sigma}\right)\left(\left[g \sigma^{-1}\right]_{H_{\sigma}}\right) & =\left.(D \iota)\right|_{[g]_{H}}\left(\left(p_{*} \pi_{\sigma}\right)\left([g]_{H}\right)\right) \\
& =\left.(D \iota)\right|_{[g]_{H}}\left(\left.(D p)\right|_{g}\left(\pi_{\sigma}(g)\right)\right) \\
& =\left.(D \iota)\right|_{[g]_{H}}\left(\left.(D p)\right|_{g}\left(R_{\sigma} \pi\left(g \sigma^{-1}\right)\right)\right) \\
& =\left.D\left(\iota \circ p \circ R_{\sigma}\right)\right|_{g \sigma^{-1}}\left(\pi\left(g \sigma^{-1}\right)\right) \\
& =\left.D\left(p_{\sigma}\right)\right|_{g \sigma^{-1}}\left(\pi\left(g \sigma^{-1}\right)\right)=\left(\left(p_{\sigma}\right)_{*} \pi\right)\left(\left[g \sigma^{-1}\right]_{H_{\sigma}}\right)
\end{aligned}
$$

for any $[g]_{H} \in G / H$ and the claim follows.

\subsection{Foliation Point of View.}

It is somewhat unexpected that the $\pi$-coisotropy of a conjugate subgroup $\operatorname{Ad}_{\sigma} H$ is not related to the $\operatorname{Ad}_{\sigma}(\pi)$-coisotropy of the subgroup $H$ but related to the $R_{\sigma} \pi$-coisotropy of $H$. In this section, we use a foliation viewpoint to give a more conceptual explanation of this phenomenon. We call a foliation $\mathcal{F}$ on a manifold $M$ regular if the leaf (i.e. the quotient) space $M / \mathcal{F}$ inherits a well-defined manifold structure from $M$.

Let $\mathcal{F}$ be a regular foliation on a manifold $M$ and $\rho \in \wedge^{k} T M$ be a tensor field on $M$. We call $\mathcal{F} \rho$-coisotropic if for any element $[\eta]$ of the holonomy groupoid $\mathfrak{G}$ that goes from $s \in M$ to $t \in M$ (and hence $s, t$ belong to the same leaf $L$ of $\mathcal{F}$ ), there is a (leaf-preserving) local diffeomorphism $\eta$, implementing $[\eta]$, from a neighbourhood of $s$ to a neighbourhood of $t$ with $\eta(s)=t$, such that

$$
(D \eta)_{s}(\rho(s))-\rho(t) \in T_{t} L \wedge\left(\wedge^{k-1} T_{t} M\right),
$$

and hence, $\rho$ projects to a well-defined tensor field $[\rho]=\rho / \mathcal{F}$ on $M / \mathcal{F}$. Note that the differential $D \eta$ of $\eta$ is a local vector bundle map from $T \mathcal{F}$ to $T \mathcal{F}$, where $T \mathcal{F}=\cup_{L \in \mathcal{F}} T L$.

Fix a tensor field $\pi$ on $G$. We consider the category $\mathcal{C}$ of $G$-manifolds $M$ endowed with $\pi$-covariant tensor field $\rho$ and a regular $\rho$-coisotropic foliation $\mathcal{F}$ that is invariant under the $G$-action on $M$. A morphism between two 
$\operatorname{objects}(M, \rho, \mathcal{F})$ and $(\tilde{M}, \tilde{\rho}, \tilde{\mathcal{F}})$ is a smooth $G$-equivariant map $\phi: M \rightarrow \tilde{M}$, i.e. $\phi(g x)=g \phi(x)$ for all $(g, x) \in G \times M$, that induces a well-defined smooth $\operatorname{map}[\phi]: M / \mathcal{F} \rightarrow \tilde{M} / \tilde{\mathcal{F}}$ and sends the tensor field $\rho$ on $M$ to $\tilde{\rho}$ on $M$, i.e. $(D \phi)(\rho(x))=\tilde{\rho}(\phi(x))$ for all $x \in M$.

It is easily recognized that the map $[\phi]$ induced by such a morphism $\phi$ is automatically $G$-equivariant and sends $[\rho]$ to $[\tilde{\rho}]$. It is natural to see that for a given object $(M, \rho, \mathcal{F})$ of $\mathcal{C}$, any diffeomorphism $\phi: M \rightarrow M$ produces an object $(\tilde{M}, \tilde{\rho}, \tilde{\mathcal{F}})$ of $\mathcal{C}$ with $\tilde{M}=M, \tilde{\mathcal{F}}=\phi_{*} \mathcal{F}$ whose leaves are exactly the images of leaves of $\mathcal{F}$ under $\phi$, and $\tilde{\rho}=(D \phi)(\rho)$, such that $\phi$ becomes an invertible morphism from $(M, \rho, \mathcal{F})$ to $(\tilde{M}=M, \tilde{\rho}, \tilde{\mathcal{F}})$. In particular, $\tilde{\rho}=(D \phi)(\rho)$ is $\pi$-covariant just like $\rho$, and $\tilde{\mathcal{F}}=\phi_{*} \mathcal{F}$ is $\tilde{\rho}$-coisotropic.

For each connected closed subgroup $H$ of $G$, the $G$-manifold $G$ has a regular foliation $\mathcal{F}_{H}$ with the right cosets $g H, g \in G$, as leaves, such that each holonomy groupoid element $[\eta]$ is implemented by a right translation $R_{h}$ with $h \in H$, which implies that for any tensor field $\rho$ on $G, \mathcal{F}_{H}$ is $\rho$ coisotropic if and only if the subgroup $H$ is $\rho$-coisotropic. Note that the diffeomorphism $R_{\sigma}: G \rightarrow G$ with $\sigma \in G$ maps the foliation $\mathcal{F}_{H}$ determined by $H$ to the foliation $\mathcal{F}_{\mathrm{Ad}_{\sigma^{-1}} H}$ determined by $\operatorname{Ad}_{\sigma^{-1}} H$, because it sends the leaf $g H$ of $\mathcal{F}_{H}$ to the leaf

$$
(g H) \sigma=g \sigma\left(\sigma^{-1} H \sigma\right)=g \sigma \operatorname{Ad}_{\sigma^{-1}} H
$$

of $\mathcal{F}_{\mathrm{Ad}_{\sigma^{-1}} H}$ for all $g \in G$. Thus, $R_{\sigma}$ determines an invertible morphism from $\left(G, \rho, \mathcal{F}_{H}\right)$ to $\left(G, R_{\sigma} \rho, \mathcal{F}_{\mathrm{Ad}_{\sigma^{-1}} H}\right)$ for any tensor field $\rho$ on $G$ that makes the subgroup $H \rho$-coisotropic. (This means that under the diffeomorphism $R_{\sigma}$, the tensor field $\rho$ corresponds to $R_{\sigma} \rho$ while the subgroup $H$ corresponds to $\operatorname{Ad}_{\sigma^{-1}} H$, not $R_{\sigma} H$ which is not a subgroup.)

In particular, $\operatorname{Ad}_{\sigma^{-1}} H$ is $R_{\sigma} \rho$-coisotropic when $H$ is $\rho$-coisotropic. Since $R_{\sigma}$ is invertible, we have $\operatorname{Ad}_{\sigma^{-1}} H$ is $R_{\sigma} \rho$-coisotropic if and only if $H$ is $\rho$-coisotropic. Substituting $H$ by $\operatorname{Ad}_{\sigma} H$, we can also say that $H$ is $R_{\sigma} \rho$ coisotropic if and only if $\operatorname{Ad}_{\sigma} H$ is $\rho$-coisotropic. Furthermore, from the above general discussion, it is also clear why the diffeomorphism $R_{\sigma}$ induces a Poisson diffeomorphism

$$
\left(G / H=G / \mathcal{F}_{H}, \rho / \mathcal{F}_{H}\right) \rightarrow\left(G / \operatorname{Ad}_{\sigma^{-1}} H=G / \mathcal{F}_{\mathrm{Ad}_{\sigma^{-1}} H}, \rho / \mathcal{F}_{\mathrm{Ad}_{\sigma^{-1}} H}\right)
$$




\section{Poisson Grassmannians.}

\subsection{Coisotropic Subgroups in Standard $S U(n)$.}

Let us now restrict ourselves to the group $S U(n)$ and fix the embedding of $S(U(n-m) \times U(m))$ in $S U(n)$ given by:

$$
(A, B) \hookrightarrow\left(\begin{array}{cc}
A & \underline{0} \\
\underline{0} & B
\end{array}\right)
$$

Recall that the standard Poisson-Lie tensor on $S U(n)$ is defined, up to a constant factor by the Poisson 2-tensor

$$
\pi(g)=L_{g} r-R_{g} r
$$

where $r \in \mathfrak{g} \wedge \mathfrak{g}, \mathfrak{g}=\mathfrak{s u}(n)$ in the following, is the $r$-matrix given by

$$
r=\sum_{1 \leq i<j \leq n} X_{i j}^{+} \wedge X_{i j}^{-}
$$

Here, we are considering the Cartan decomposition of $\mathfrak{g}$ determined by the subalgebra of diagonal matrices and denote by $X_{i j}^{ \pm}$the corresponding root vectors

$$
X_{i j}^{+}=\imath\left(e_{i j}+e_{j i}\right) \quad X_{i j}^{-}=e_{i j}-e_{j i}
$$

with $e_{i j}$ denoting a standard matrix unit.

It is then easily seen that $S(U(m) \times U(n-m))$ is a Poisson-Lie subgroup in $S U(n)$. We will denote its Lie algebra by $\mathfrak{s}(\mathfrak{u}(n-m) \times \mathfrak{u}(m))$.

Proposition 3.1. Let $m \leq\left[\frac{n}{2}\right]$ with $4 \leq n \in \mathbb{N}, c \in[0,1]$, and let

$$
\begin{array}{r}
\sigma(c, m)=\sqrt{c} \sum_{i=1}^{m} e_{i i}+\sum_{i=m+1}^{n-m} e_{i i}+\sqrt{c} \sum_{i=n-m+1}^{n} e_{i i} \\
+\sqrt{1-c} \sum_{i=1}^{m}\left(e_{n+1-i, i}-e_{i, n+1-i}\right) .
\end{array}
$$

Then, subgroup $A d_{\sigma(c, m)}(U(n-m) \times U(m))$ is coisotropic in $U(n)$. Analogously $\operatorname{Ad}_{\sigma(c, m)} S(U(n-m) \times U(m))$ is coisotropic in the standard $S U(n)$. 
Proof. Let $\sigma=\sigma(c, m)$ throughout the proof. Then,

$$
\begin{aligned}
\sigma^{-1}=\sqrt{c}( & \left.\sum_{i=1}^{m} e_{i, i}+e_{n+1-i, n+1-i}\right) \\
& +\sum_{k=m+1}^{n-m} e_{k, k}-\sqrt{1-c}\left(\sum_{i=1}^{m} e_{n+1-i, i}-e_{i, n+1-i}\right) .
\end{aligned}
$$

As in the proof of Theorem 3 of [20], it suffices to show that

$$
\left(A d_{\sigma^{-1}} r\right)-(2 c-1) r \in(\mathfrak{u}(n-m) \times \mathfrak{u}(m)) \wedge \mathfrak{u}(n) .
$$

Let $A=\sqrt{c(1-c)}$. First of all we remark that the following relations hold true:

$$
\begin{aligned}
\sigma^{-1} e_{i, j} \sigma & =c e_{i, j}+(1-c) e_{n+1-i, n+1-j}-A\left(e_{i, n+1-j}+e_{n+1-i, j}\right) \\
\sigma^{-1} e_{n+1-j, n+1-i} \sigma & =c e_{n+1-j, n+1-i}+(1-c) e_{j, i}+A\left(e_{n+1-j, i}+e_{j, n+1-i}\right) \\
\sigma^{-1} e_{i, n+1-j} \sigma & =c e_{i, n+1-j}-(1-c) e_{n+1-i, j}+A\left(e_{i, j}-e_{n+1-i, n+1-j}\right) \\
\sigma^{-1} e_{n+1-i, j} \sigma & =c e_{n+1-i, j}-(1-c) e_{i, n+1-j}+A\left(e_{i, j}-e_{n+1-i, n+1-j}\right)
\end{aligned}
$$

for every $1 \leq i, j \leq m$. Furthermore:

$$
\begin{aligned}
\sigma^{-1} e_{i, m+p} \sigma & =\sqrt{c} e_{i, m+p}-\sqrt{1-c} e_{n+1-i, m+p} \\
\sigma^{-1} e_{n+1-i, m+p} \sigma & =\sqrt{c} e_{n+1-i, m+p}+\sqrt{1-c} e_{i, m+p} \\
\sigma^{-1} e_{m+p, i} \sigma & =\sqrt{c} e_{m+p, i}-\sqrt{1-c} e_{m+p, n+1-i} \\
\sigma^{-1} e_{m+p, n+1-i} \sigma & =\sqrt{c} e_{m+p, n+1-i}+\sqrt{1-c} e_{m+p, i}
\end{aligned}
$$

for every $1 \leq i \leq m$ and $1 \leq p \leq n-2 m$. Lastly:

$$
\sigma^{-1} e_{i, j} \sigma=e_{i, j}
$$

when $m+1 \leq i, j \leq n-m$. From these equalities one can compute:

$$
\sigma^{-1} X_{i, n+1-j}^{ \pm} \sigma=c X_{i, n+1-j}^{ \pm} \mp(1-c) X_{j, n+1-i}^{ \pm}+A\left(X_{i, j}^{ \pm} \mp X_{n+1-j, n+1-i}^{ \pm}\right)
$$

for all $1 \leq i \neq j \leq m$, and

$$
\begin{aligned}
\sigma^{-1} X_{i, j}^{ \pm} \sigma=c X_{i, j}^{ \pm}-A X_{i, n+1-j}^{ \pm} \mp A X_{j, n+1-i}^{ \pm} \\
\pm(1-c) X_{n+1-j, n+1-i}^{ \pm} \\
\sigma^{-1} X_{n+1-j, n+1-i}^{ \pm} \sigma=c X_{n+1-j, n+1-i}^{ \pm} \pm(1-c) X_{i, j}^{ \pm} \\
\pm A X_{i, n+1-j}^{ \pm}+A X_{j, n+1-i}^{ \pm} \\
\sigma^{-1} X_{i, n+1-i}^{-} \sigma=X_{i, n+1-i}^{-} \\
\sigma^{-1} X_{i, n+1-i}^{+} \sigma=(2 c-1) X_{i, n+1-i}^{+}+2 A K_{i}
\end{aligned}
$$


for every $1 \leq i<j \leq m$, where $K_{i}=\imath\left(e_{i, i}-e_{n+1-i, n+1-i}\right)$. Furthermore:

$$
\begin{aligned}
\sigma^{-1} X_{i, m+p}^{ \pm} \sigma & =\sqrt{c} X_{i, m+p}^{ \pm} \mp \sqrt{1-c} X_{m+p, n+1-i}^{ \pm} \\
\sigma^{-1} X_{m+p, n+1-i}^{ \pm} \sigma & =\sqrt{c} X_{m+p, n+1-i}^{ \pm} \pm \sqrt{1-c} X_{i, m+p}^{ \pm}
\end{aligned}
$$

for every $1 \leq i \leq m$ and $1 \leq p \leq n-2 m$. Lastly:

$$
\sigma^{-1} X_{m+p, m+q}^{ \pm} \sigma=X_{m+p, m+q}^{ \pm}
$$

when $1 \leq p<q \leq n-2 m$.

Let's now move to $A d_{\sigma^{-1}} r$ which we divide into three separate pieces:

$$
A d_{\sigma^{-1}} r=A d_{\sigma^{-1}}(\Phi+\Theta+\Omega)
$$

where:

$$
\begin{aligned}
\Phi & =\sum_{m+1 \leq i, j \leq n-m} X_{i, j}^{+} \wedge X_{i, j}^{-} \\
\Theta= & \sum_{p=1}^{n-2 m} \sum_{i=1}^{m} X_{i, m+p}^{+} \wedge X_{i, m+p}^{-}+X_{m+p, n+1-i}^{+} \wedge X_{m+p, n+1-i}^{-} \\
& +\sum_{i=1}^{m} X_{i, n+1-i}^{+} \wedge X_{i, n+1-i}^{-} \\
\Omega & \sum_{1 \leq i<j \leq m} X_{i, j}^{+} \wedge X_{i, j}^{-}+\sum_{1 \leq i \neq j \leq m} X_{i, n+1-j}^{+} \wedge X_{i, n+1-j}^{-} \\
& \quad+\sum_{1 \leq i<j \leq m} X_{n+1-j, n+1-i}^{+} \wedge X_{n+1-j, n+1-i}^{-}
\end{aligned}
$$

By a straightforward computation, we get

$$
\begin{aligned}
A d_{\sigma^{-1}}(\Phi)= & \in(2 c-1) \Phi+[(\mathfrak{u}(n-m) \times \mathfrak{u}(m)) \wedge \mathfrak{u}(n)] \\
A d_{\sigma^{-1}}(\Theta)= & (2 c-1) \Theta+2 A \sum_{i, m+p}\left[K_{i} \wedge X_{i, n+1-i}^{-}+X_{m+p, n+1-i}^{+} \wedge X_{m}^{-}\right. \\
& \left.\quad-X_{m+p, n+1-i}^{+} \wedge X_{i, m+p}^{-}\right] \\
& \in(2 c-1) \Theta+[(\mathfrak{u}(n-m) \times \mathfrak{u}(m)) \wedge \mathfrak{u}(n)]
\end{aligned}
$$

since $K_{i}, X_{i, m+p}^{ \pm}, \Phi \in \mathfrak{u}(n-m) \times \mathfrak{u}(m)$. The computation of $A d_{\sigma^{-1}}(\Omega)$ is much more tedious. It involves 


$$
\begin{array}{r}
A d_{\sigma^{-1}}(\Omega)= \\
\sum_{1 \leq i<j \leq m}\left(c X_{i, j}^{+}-A X_{i, n+1-j}^{+}-A X_{j, n+1-i}^{+}+(1-c) X_{n+1-j, n+1-i}^{+}\right) \\
\wedge\left(c X_{i, j}^{-}-A X_{i, n+1-j}^{-}+A X_{j, n+1-i}^{-}-(1-c) X_{n+1-j, n+1-i}^{-}\right) \\
+\sum_{1 \leq i \neq j \leq m}\left(A X_{i, j}^{+}+c X_{i, n+1-j}^{+}-(1-c) X_{j, n+1-i}^{+}-A X_{n+1-j, n+1-i}^{+}\right) \\
\wedge\left(A X_{i, j}^{-}+c X_{i, n+1-j}^{-}+(1-c) X_{j, n+1-i}^{-}+A X_{n+1-j, n+1-i}^{-}\right) \\
+\sum_{1 \leq i<j \leq m}\left((1-c) X_{i, j}^{+}+A X_{i, n+1-j}^{+}+A X_{j, n+1-i}^{+}+c X_{n+1-j, n+1-i}^{+}\right) \\
\wedge\left(-(1-c) X_{i, j}^{-}-A X_{i, n+1-j}^{-}+A X_{j, n+1-i}^{-}+c X_{n+1-j, n+1-i}^{-}\right) .
\end{array}
$$

The sum of all the wedge products of a "+ corresponding "--term" on the right of $\wedge$ is $(2 c-1) \Omega$. All the remaining wedge products of a term on the left of $\wedge$ and a term on the right of $\wedge$ are in $(\mathfrak{u}(n-m) \times \mathfrak{u}(m)) \wedge u(n)$, except for those involving the products $X_{i, n+1-j}^{+} \wedge X_{j, n+1-i}^{+}$since multiples of $X_{i, n+1-j}^{+}$and $X_{j, n+1-i}^{+}$are the only terms not in the Lie subalgebra $u(n-m) \times u(m)$. It is easy to check that the sum of all those wedge products involving $X_{i, n+1-j}^{+} \wedge X_{j, n+1-i}^{+}$is 0 . So we get

$$
A d_{\sigma^{-1}}(\Omega) \in(2 c-1) \Omega+[(\mathfrak{u}(n-m) \times \mathfrak{u}(m)) \wedge \mathfrak{u}(n)]
$$

Putting all together, we have that:

$$
A d_{\sigma^{-1}} r=(2 c-1) r+[(\mathfrak{u}(n-m) \times \mathfrak{u}(m)) \wedge \mathfrak{u}(n)]
$$

as wanted.

We will denote with $\tau_{\sigma_{c}}$ the projected Poisson 2-tensor on the complex Grassmannian $G_{n}^{m} \mathbb{C}=S U(n) / S(U(m) \times U(n-m))$.

\subsection{Covariance of Tensor Structures.}

We plan now to describe a general argument which shows that the Poisson pencil generated by the only (up to constant) $S U(n)$-invariant Poisson structure on $G_{m}^{n} \mathbb{C}$ together with any $\tau_{\sigma_{c}}$ covers all of $S U(n)$-covariant Poisson structures on the complex Grassmannians. 
Let $M$ be a $G$-manifold. Given two tensor fields $\pi$ and $\rho$ (of the same kind) on $G$ and $M$ respectively, $\rho$ is called $\pi$-covariant if (the differential $D \mu$ of) the action

$$
\mu:(g, h) \in G \times M \mapsto g h \in M
$$

sends the product tensor $\pi \times \rho$ on $G \times M$ to $\rho$ on $M$. When $\pi, \rho$ are Poisson 2-tensors this means that $\mu$ is a Poisson map (w.r.t. $\pi \times \rho$ and $\rho$ ), where the product tensor $\pi \times \rho$ on $G \times M$ is defined by

$$
(\pi \times \rho)(g, h):=\pi(g) \oplus \rho(h) \in \wedge^{2} T_{g} G \oplus \wedge^{2} T_{h} M \subset \wedge^{2} T_{(g, h)}(G \times M) .
$$

The general condition can be summarized as

$$
(D \mu)(\pi \oplus \rho)=\rho .
$$

It is interesting to note that a tensor field $\rho$ on a $G$-manifold $M$ is $G$-invariant if and only if $\rho$ is 0 -covariant for the vanishing tensor field 0 on $G$, i.e. the action operation

$$
\mu:(g, h) \in G \times M \mapsto g h \in M
$$

sends the product tensor $0 \times \rho$ on $G \times M$ to $\rho$ on $M$, because for all $\left(g_{0}, h_{0}\right) \in$ $G \times M$,

$$
\begin{aligned}
& (D \mu)_{\left(g_{0}, h_{0}\right)}\left((0 \times \rho)\left(g_{0}, h_{0}\right)\right)=(D \mu)_{\left(g_{0}, h_{0}\right)}\left(0 \oplus \rho\left(h_{0}\right)\right) \\
& =\left(\frac{\partial \mu}{\partial g}\right)_{\left(g_{0}, h_{0}\right)}(0)+\left(\frac{\partial \mu}{\partial h}\right)_{\left(g_{0}, h_{0}\right)}\left(\rho\left(h_{0}\right)\right)=L_{g_{0}}\left(\rho\left(h_{0}\right)\right)
\end{aligned}
$$

and hence,

$$
(D \mu)_{\left(g_{0}, h_{0}\right)}\left((0 \times \rho)\left(g_{0}, h_{0}\right)\right)=\rho\left(g_{0} h_{0}\right)
$$

if and only if

$$
L_{g_{0}}\left(\rho\left(h_{0}\right)\right)=\rho\left(g_{0} h_{0}\right) .
$$

Note that the multiplicativity of a tensor field $\pi$ on $G$ is equivalent to the condition that $\pi$ is $\pi$-covariant, i.e. the multiplication operation

$$
\mu:(g, h) \in G \times G \mapsto g h \in G
$$

sends the product tensor $\pi \times \pi$ on $G \times G$ to $\pi$ on $G$, because for all $\left(g_{0}, h_{0}\right) \in$ $G \times G$,

$$
\begin{aligned}
& (D \mu)_{\left(g_{0}, h_{0}\right)}\left((\pi \times \pi)\left(g_{0}, h_{0}\right)\right)=(D \mu)_{\left(g_{0}, h_{0}\right)}\left(\pi\left(g_{0}\right) \oplus \pi\left(h_{0}\right)\right) \\
& =\left(\frac{\partial \mu}{\partial g}\right)_{\left(g_{0}, h_{0}\right)}\left(\pi\left(g_{0}\right)\right)+\left(\frac{\partial \mu}{\partial h}\right)_{\left(g_{0}, h_{0}\right)}\left(\pi\left(h_{0}\right)\right) \\
& =R_{h_{0}}\left(\pi\left(g_{0}\right)\right)+L_{g_{0}}\left(\pi\left(h_{0}\right)\right)
\end{aligned}
$$


and hence,

$$
(D \mu)_{\left(g_{0}, h_{0}\right)}\left((\pi \times \pi)\left(g_{0}, h_{0}\right)\right)=\pi\left(g_{0} h_{0}\right)
$$

if and only if

$$
R_{h_{0}}\left(\pi\left(g_{0}\right)\right)+L_{g_{0}}\left(\pi\left(h_{0}\right)\right)=\pi\left(g_{0} h_{0}\right) .
$$

Similarly, the affinity of a tensor field $\rho$ on $G$ is equivalent to the condition that $\rho$ is $\pi$-covariant for the field $\pi=\rho_{l}:=\rho-(\rho(e))^{l}$ (which is multiplicative when $\rho$ is indeed affine), i.e. the multiplication operation

$$
\mu:(g, h) \in G \times G \mapsto g h \in G
$$

sends the product tensor $\pi \times \rho$ on $G \times G$ to $\rho$ on $G$, because for all $\left(g_{0}, h_{0}\right) \in$ $G \times G$,

$$
\begin{gathered}
(D \mu)_{\left(g_{0}, h_{0}\right)}\left((\pi \times \rho)\left(g_{0}, h_{0}\right)\right)=(D \mu)_{\left(g_{0}, h_{0}\right)}\left(\pi\left(g_{0}\right) \oplus \rho\left(h_{0}\right)\right) \\
=\left(\frac{\partial \mu}{\partial g}\right)_{\left(g_{0}, h_{0}\right)}\left(\pi\left(g_{0}\right)\right)+\left(\frac{\partial \mu}{\partial h}\right)_{\left(g_{0}, h_{0}\right)}\left(\rho\left(h_{0}\right)\right)=R_{h_{0}}\left(\pi\left(g_{0}\right)\right)+L_{g_{0}}\left(\rho\left(h_{0}\right)\right) \\
=R_{h_{0}}\left(\rho\left(g_{0}\right)\right)-R_{h_{0}}\left(L_{g_{0}}(\rho(e))\right)+L_{g_{0}}\left(\rho\left(h_{0}\right)\right)
\end{gathered}
$$

and hence,

$$
(D \mu)_{\left(g_{0}, h_{0}\right)}\left((\pi \times \rho)\left(g_{0}, h_{0}\right)\right)=\rho\left(g_{0} h_{0}\right)
$$

if and only if

$$
R_{h_{0}}\left(\pi\left(g_{0}\right)\right)+L_{g_{0}}\left(\rho\left(h_{0}\right)\right)-R_{h_{0}}\left(L_{g_{0}}(\rho(e))\right)=\rho\left(g_{0} h_{0}\right) .
$$

We give an interesting application of the above viewpoint. First, we give a proof of the following known general result, using the concept discussed above.

Proposition 3.2. Let $\pi \in \wedge^{k} T G$ and $M$ be a G-manifold. If $\rho \in \wedge^{k} T M$ is $\pi$-covariant and $\tilde{\rho} \in \wedge^{k} T M$ is $G$-invariant, then $\rho+\tilde{\rho}$ (or any tensor in $\rho+\mathbb{R} \tilde{\rho})$ is $\pi$-covariant and the Schouten bracket $[[\rho, \tilde{\rho}]]$ (or any tensor in $[[\rho, \mathbb{R} \tilde{\rho}]])$ is $G$-invariant.

Proof. The given conditions can be summarized as

$$
(D \mu)(\pi \oplus \rho)=\rho \text { and }(D \mu)(0 \oplus \tilde{\rho})=\tilde{\rho}
$$

for the action map $\mu: G \times M \rightarrow M$. Clearly, we have

$$
(D \mu)(\pi \oplus(\rho+\tilde{\rho}))=(D \mu)((\pi \oplus \rho)+(0 \oplus \tilde{\rho}))=\rho+\tilde{\rho}
$$


which means that $\rho+\tilde{\rho}$ is $\pi$-covariant. On the other hand, we first note that the Schouten bracket

$$
[[\kappa \oplus 0,0 \oplus \lambda]]=0 \text { in } \wedge^{k} T(G \times M)
$$

for any tensor $\kappa \in \wedge^{k} T G$ and $\lambda \in \wedge^{k} T M$. Now since the differential $D \mu$ preserves the Schouten bracket operation, we also have

$$
\begin{gathered}
{[[\rho, \tilde{\rho}]]=[[(D \mu)(\pi \oplus \rho),(D \mu)(0 \oplus \tilde{\rho})]]=(D \mu)([[\pi \oplus \rho, 0 \oplus \tilde{\rho}]])} \\
=(D \mu)([[\pi, 0]] \oplus[[\rho, \tilde{\rho}]])=(D \mu)(0 \oplus[[\rho, \tilde{\rho}]])
\end{gathered}
$$

which means that $[[\rho, \tilde{\rho}]]$ is $G$-invariant.

For Poisson tensors $\rho, \tilde{\rho}$ on $M$ (i.e. $[[\rho, \rho]]=0=[[\tilde{\rho}, \tilde{\rho}]]$ ), the sum $\rho+\tilde{\rho}$ is Poisson if and only if $[[\rho, \tilde{\rho}]]=0$. If $\rho \in \wedge^{2} T M$ is a $\pi$-covariant Poisson tensor and $\tilde{\rho} \in \wedge^{2} T M$ is $G$-invariant Poisson tensor, then $\rho+\tilde{\rho}$ (or any tensor in $\rho+\mathbb{R} \tilde{\rho})$ is a $\pi$-covariant Poisson tensor if there is no non-trivial $G$-invariant 3 -tensor on $M$. For any compact symmetric space this last condition is equivalent to $H_{D R}^{3}(M)=0$ which is verified, for example, when $M=G_{m}^{n} \mathbb{C}$. This proves that if $\rho$ is the $S U(n)$ invariant Poisson tensor on $G_{m}^{n} \mathbb{C}$ then $\rho$ and $\tau_{c}$ are compatible $\left(\left[\left[\rho, \tau_{c}\right]\right]=0\right)$ and therefore generates the Poisson pencil of $S U(n)$-covariant Poisson tensors.

In particular, if $X \in \wedge^{2} \mathfrak{g}$ and $X^{l}$ is a (of course $G$-invariant) Poisson 2-tensor on $G$, then $\rho+X^{l}$ is an affine Poisson 2-tensor on $G$ (which is also $\rho_{l}$-covariant and hence, $\left.\left(\rho+X^{l}\right)_{l}=\rho_{l}\right)$ for any affine Poisson 2-tensor $\rho$ on $G$ (which is $\rho_{l}$-covariant for the multiplicative $\left.\rho_{l}:=\rho-(\rho(e))^{l}\right)$.

\subsection{Lagrangian Subalgebras.}

In [8] Drinfel'd showed how to relate Poisson homogeneous spaces of a given Poisson-Lie group to orbits (under a natural action) of the group itself on the algebraic variety $\mathcal{L}$ of Lagrangian subalgebra of the double $D(\mathfrak{g})$. Such construction led Karolinsky [11] to a classification of Poisson homogeneous spaces - at least when $D(\mathfrak{g})$ is complex semisimple - in terms of combinatorial data associated to the root system. Later on Evens and $\mathrm{Lu}$ in [9] showed how to define a natural Poisson bivector on $\mathcal{L}$ such that the Drinfel'd map is always an equivariant Poisson map. In this context a quotient by a coisotropic subgroup corresponds to orbits in $\mathcal{L}$ containing at least one split subalgebra. In this paragraph we will describe such Lagrangian subalgebras 
for our specific family of covariant Poisson brackets on complex Grassmannians, generalizing results in [20]. We give a direct proof of the following lemma which can also be deduced from [14].

Lemma 3.3. Let $G$ be a Poisson-Lie group, $H$ a closed connected subgroup, with Lie algebra $\mathfrak{h} \subseteq \mathfrak{g}$. Let $\sigma \in G$ be such that $\mathfrak{h}_{\sigma}=A d_{\sigma} \mathfrak{h}$ is coisotropic in $\mathfrak{g}$ (i.e. $\left.\left.(d \pi)\right|_{\mathfrak{h}_{\sigma}} \subseteq \mathfrak{h}_{\sigma} \wedge \mathfrak{g}\right)$. Then, the Lagrangian subalgebras corresponding to the Poisson structure $\tau_{\sigma}$ on the homogeneous space $G / H$ over the point $x_{0}:=e H$ is

$$
\left.\mathfrak{h}+\left\{(x, \beta) \in \mathfrak{g} \times \mathfrak{g}^{*} \mid \beta \in \mathfrak{h}^{\perp}, \tilde{\pi}_{\sigma^{-1}}\right\lrcorner \beta=x\right\}
$$

Proof. By construction (see [8]) the Lagrangian subalgebra over the point $\sigma \cdot x_{0}$ is split and equals $\mathfrak{l}=\mathfrak{h}_{\sigma} \oplus \mathfrak{h}_{\sigma}^{\perp}$. We will use $G$-equivariance of the correspondence between points and Lagrangian subalgebras. Recall, that the action of $G$ on its double $D(\mathfrak{g}) \simeq \mathfrak{g} \oplus \mathfrak{g}^{*}$ is given by

$$
g \cdot(X, \alpha)=\left(A d_{g} X+\tilde{\pi}(g)\left(A d_{g}^{*} \alpha,-\right), A d_{g}^{*} \alpha\right)
$$

Therefore, letting $g=\sigma^{-1}$ act on $\left(A d_{\sigma} Y, A d_{\sigma}^{*} \beta\right)$, we get

$$
\left.\sigma \cdot \mathfrak{l}=\mathfrak{h} \oplus\left\{(x, \beta) \in \mathfrak{g} \times \mathfrak{g}^{*} \mid \beta \in \mathfrak{h}^{\perp}, \tilde{\pi}_{\sigma^{-1}}(e)\right\lrcorner \beta=x\right\}
$$

Note that the Lagrangian subalgebra $\sigma \cdot \mathfrak{l}$ is exactly the Lagrangian subalgebra complementary to $\mathfrak{g}$ associated with the affine Poisson bracket $\pi_{\sigma}$ (see [13])

The Lagrangian subalgebras corresponding to Poisson homogeneous complex Grassmannians, over the point $x_{0}=e H$ can be computed either by solving

$$
\left.\tilde{\pi}_{\sigma^{-1}}(e)\right\lrcorner \beta=x
$$

or remarking that $\mathfrak{h}_{\sigma}^{\perp}$ is generated, as a vector space, by the following elements

$$
\begin{aligned}
& \left\langle\mp x_{ \pm}^{i j}+x_{ \pm}^{n+1-j, n+1-i}-\frac{2 c-1}{\sqrt{c(1-c)}} x_{ \pm}^{j, n+1-i} \quad, 1 \leq i<j \leq m\right\rangle \\
& \left\langle x_{ \pm}^{i, n+1-j}+\mp x_{ \pm}^{j, n+1-i} \quad, 1 \leq i<j \leq m\right\rangle \\
& \left\langle\sqrt{c} x_{ \pm}^{i, m+p} \pm \sqrt{1-c} x_{ \pm}^{m+p, n+1-i} \quad, 1 \leq i \leq m, 1 \leq p \leq n-2 m\right\rangle \\
& \left\langle x_{-}^{i, n+1-i}, h_{i}+h_{n-i}+\frac{2 c-1}{\sqrt{c(1-c)}} \sum_{j=n-i+1}^{n} x_{+}^{n+1-j, j} \quad, 1 \leq i \leq m\right\rangle
\end{aligned}
$$


where $x_{ \pm}^{h k}$ are the dual elements of $X_{h k}^{ \pm}$and $h_{l}$ are the dual elements of the Cartan subalgebra standard basis $H_{l}=\imath\left(e_{l, l}-e_{n, n}\right)$.

Remark that $\mathfrak{h}_{\sigma}^{\perp}$ is a Lie subalgebra of $\mathfrak{g}^{*}$ and, as such, can be integrated to a coisotropic subgroup $H^{\perp}$ of $G^{*}$. The Poisson homogeneous space $G^{*} / H^{\perp}$ is called the complementary dual of $G / H$ in [4] where it is shown that it fits into a quantum duality scheme.

\section{Poisson Embeddings.}

\subsection{General Embeddings.}

Lemma 4.1. Let $(G, \pi)$ be a Poisson-Lie group (with Lie cobracket $\delta$ ). Let $K$ be a closed Poisson-Lie subgroup and let $H^{\prime}$ be a closed coisotropic subgroup in $G$. Then, $H=K \cap H^{\prime}$ is a coisotropic subgroup of $K$ and the natural map

$$
\imath: K / H \rightarrow G / H^{\prime} ;[k]_{H} \mapsto[k]_{H^{\prime}}
$$

is a Poisson embedding with respect to the projected Poisson structures. If $K \cup H^{\prime}$ generates $G$ then $K / H$ is Poisson diffeomorphic to $G / H^{\prime}$.

Proof. Coisotropy of $K \cap H^{\prime}$ in $K$ follows from its infinitesimal characterization. In fact, intersecting a subcoalgebra $\mathfrak{k}$ (i.e. $\delta(\mathfrak{k}) \subseteq \mathfrak{k} \wedge \mathfrak{k}$ ) with a subcoideal $\mathfrak{h}^{\prime}$ (i.e. $\delta\left(\mathfrak{h}^{\prime}\right) \subseteq \mathfrak{h}^{\prime} \wedge \mathfrak{g}$ ) gives a subcoideal of $\mathfrak{k}$. The map $\imath$ is the unique map such that $p^{\prime} \circ i=\imath \circ p_{H}$, where $i: K \hookrightarrow G$ is the Poisson embedding, $p^{\prime}: G \rightarrow G / H^{\prime}$ and $p_{H}: K \rightarrow K / H$ are the natural Poisson projections. It is then easily seen that $\imath$ is injective, Poisson and with injective differential. This map is also surjective if every $g \in G$ can be written as $g=k h^{\prime}$, with $h \in K$ and $h^{\prime} \in H^{\prime}$ so that the last statement follows as well.

\section{Examples:}

1. Let $K^{\prime} \subseteq K \subseteq G$ be a chain of Poisson-Lie groups. Then, the natural map from $K / K^{\prime}$ to $G / K^{\prime}$ is a Poisson embedding. In this way, for example, one can prove that standard Poisson spheres $\mathbb{S}^{2 k+1}$ (i.e. quotients $S U(k+1) / S U(k)$ w.r.t. the standard Poisson $S U(k+1))$ are embedded in standard Poisson complex Stiefel manifolds $V_{k}^{n} \mathbb{C} \simeq$ $S U(n) / S U(k)$

2. Let $H_{\sigma}$ be a 1-parameter family of coisotropic subgroups containing a Poisson-Lie group $K=H_{0}$. Then, we have a Poisson embedding from $K /\left(K \cap H_{\sigma}\right)$ to $G / H_{\sigma}$. This example will be frequently used in what follows. 
3. Let $G=S U(n)$ and let $K=S U(n-1)$ be the Poisson-Lie subgroup of lower right corner matrices (i.e. the first row and column are $(1,0, \ldots, 0)$. Then, let $H^{\prime}=S U(n-1)$ be the Poisson-Lie subgroup of upper left corner matrices. We have: $H=S U(n-2)$ and $K / H \simeq \mathbb{S}^{2 n-1}$ with the standard Poisson structure, which is, then, naturally embedded in $S U(n) / S U(n-1) \simeq \mathbb{S}^{2 n+1}$. Taking $H_{p}=S U(n-p)$ as upper left corner matrices and repeating the argument, we find a chain of Poisson embeddings of spheres explaining the symplectic foliation of the standard Poisson spheres.

We will now give a description of Poisson embeddings for standard complex projective spaces and complex Grassmannians and see how it relates with the Bruhat-Poisson foliation. The same argument will then be generalized to non standard complex Grassmannians (and projective spaces) in what follows.

\subsection{Complex Projective Spaces.}

In this section, the idea is to explain how the subgroup method can be used to describe (part of) the symplectic foliation both for standard and non standard complex projective spaces. Let us recall that from the classification of Poisson-Lie subgroups of a given standard compact Poisson-Lie group (see [21]) one can deduce that maximal Poisson-Lie subgroups in $S U(n)$ are the diagonally embedded $S(U(k) \times U(n-k)), k=1, \ldots, n$.

Let us start with the standard case. The complex projective space $\mathbb{P}^{n} \mathbb{C}$ is identified with the quotient $S U(n) / S(U(1) \times U(n-1))$ via the projection

$$
\begin{aligned}
& p: S U(n) \rightarrow \mathbb{P}^{n-1} \mathbb{C} \\
& A \mapsto\left[A \cdot{ }^{t}(0, \ldots, 0,1)\right]=\left[A^{(n)}\right]
\end{aligned}
$$

where $A^{(i)}$ denotes the $i^{t h}$-column of the matrix $A$ and ${ }^{t}(0, \ldots, 0,1)$ the transposed column vector. The corresponding standard Poisson structure has symplectic foliation described by Schubert cells (see [21] for more explicit description) which is, in this case, described as a chain of embeddings

$$
\mathbb{P}^{0} \mathbb{C} \subseteq \mathbb{P}^{1} \mathbb{C} \subseteq \ldots \subseteq \mathbb{P}^{n-2} \mathbb{C} \subseteq \mathbb{P}^{n-1} \mathbb{C}
$$

each of which is given by equations $Z_{1}=\ldots Z_{k}=0$ in homogeneous coordinates. It is then easily seen that the parabolic subgroups corresponding to $S=\left\{\alpha_{1}, \ldots, \widehat{\alpha_{k}}, \ldots, \alpha_{n-1}\right\}$ intersects $S U(n)$ in a Poisson-Lie subgroup

$$
K_{k}=\left\{\left(\begin{array}{cc}
A & 0 \\
0 & B
\end{array}\right) \in S(U(k) \times U(n-k))\right\}
$$

having as image under the projection $p$ exactly $X_{k}=\mathbb{P}^{k-1} \mathbb{C}$. 
Theorem 4.2. For any $k=1, \ldots, n-2$, we have $K_{k} \cap K_{n-1} \simeq S(U(k) \times$ $U(n-k-1) \times U(1)) \simeq U(k) \times U(n-k-1)$. Furthermore, $K_{k} / K_{k} \cap K_{n-1}$ is Poisson diffeomorphic to the standard Poisson $\mathbb{P}^{n-k-1} \mathbb{C}$ and projects onto $X_{k}$ via $p$.

Proof. The statement about the intersection is easily verified. For the second statement consider the map

$$
\imath: \frac{S U(n-k)}{S(U(n-k-1) \times U(1))} \longrightarrow \frac{K_{k}}{K_{k} \cap K_{n-1}} ; \quad \imath([B])=\left[\left(\begin{array}{cc}
1 & 0 \\
0 & B
\end{array}\right)\right] .
$$

This map is a Poisson diffeomorphism due to an application of lemma 4.1 remarking that $\imath(S(U(n-k-1) \times U(1)))=K_{k} \cap S U(n-k)$, and that the union $\left(K_{n-1} \cap K_{k}\right) \cup S U(n-k)$ generates $K_{k}$.

Note that $p\left(K_{1}\right) \supseteq \ldots p\left(K_{n-2}\right) \supseteq p\left(K_{n-1}\right)=\{*\}$, i.e. all the embeddings granted by the proposition are contained one into another and overlap the Schubert cell decomposition.

Let's move to the non standard case. As we have seen in Proposition 2.2 one can consider it simply as obtained via a different projection, i.e. identifying the complex projective space with a quotient of $S U(n)$ as image of

$$
\begin{aligned}
& p_{\sigma}: S U(n) \rightarrow \mathbb{P}^{n-1} \mathbb{C} \\
& p_{\sigma}: A \mapsto\left[A \cdot{ }^{t}(\sqrt{c}, 0, \ldots, 0, \sqrt{1-c})\right]=\left[\sqrt{c} A^{(1)}+\sqrt{1-c} A^{(n)}\right] .
\end{aligned}
$$

The stabilizer, in this case, is the subgroup $H_{\sigma}=A d_{\sigma(c, 1)} U(n-1)$. Differently from the standard case, the Poisson-Lie subgroups $K_{k}$ have images which are not contained one into another. In more detail $p\left(K_{k}\right)$ consists of

$$
\begin{aligned}
{\left[\left(\begin{array}{cc}
A & 0 \\
0 & B
\end{array}\right) \cdot{ }^{t}(\sqrt{c}, 0, \ldots, 0, \sqrt{1-c})\right]=} & {\left[\left(\sqrt{c} a_{11}, \ldots, \sqrt{c} a_{k 1},\right.\right.} \\
& \left.\left.\sqrt{1-c} b_{k+1, n}, \ldots, \sqrt{1-c} b_{n, n}\right)\right]
\end{aligned}
$$

The images $X_{k}=p\left(K_{k}\right)$ satisfy, then, the equation

$$
\left\|A^{(1)}\right\|^{2}-\left\|B^{(n)}\right\|^{2}=0
$$

which, in homogeneous coordinates, can be expressed as

$$
\left|Z_{11}\right|^{2}+\ldots+\left|Z_{k 1}\right|^{2}-\frac{c}{(1-c)}\left(\left|Z_{k+1, n}\right|^{2}+\ldots+\left|Z_{n n}\right|^{2}\right)=0
$$

These are exactly the same equations for the higher dimensional singular symplectic leaves as in [12]. 
Theorem 4.3. For any $k=1, \ldots, n-1$ we have

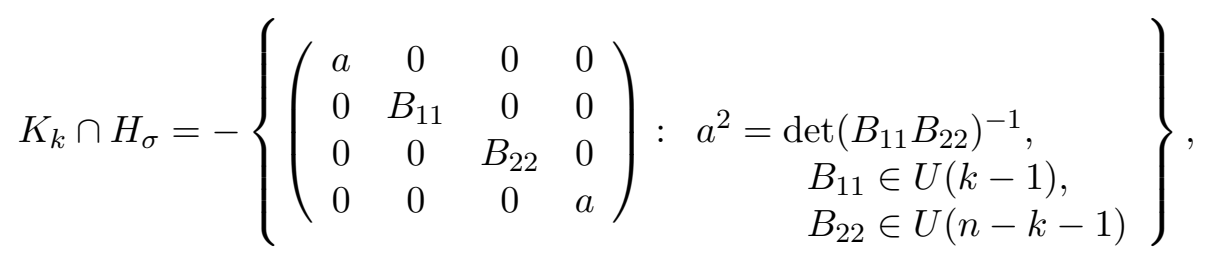

Furthermore, $K_{k} / K_{k} \cap H_{\sigma_{c}}$ is Poisson diffeomorphic to $\left(\mathbb{S}^{2 k-1} \times \mathbb{S}^{2(n-k)-1}\right) / \mathbb{T}$ (if $k=1$ to a standard Poisson odd sphere $\mathbb{S}^{2 n-3}$ ).

Proof. Let us start with $k=1$ and consider the embedding

$$
A \in U(n-1) \mapsto\left(\begin{array}{cc}
\operatorname{det}(A)^{-1} & 0 \\
0 & A
\end{array}\right) \in H:=K_{1} \subset G:=S U(n)
$$

of $U(n-1)$ ONTO the closed subgroup $H=K_{1}$ of $G=S U(n)$. Let

$$
\sigma_{c}=\left(\begin{array}{ccc}
\sqrt{c} & 0 & \sqrt{1-c} \\
0 & I_{n-2} & 0 \\
-\sqrt{1-c} & 0 & \sqrt{c}
\end{array}\right) \in S U(n)
$$

with $c \in(0,1)$. Since for any

$$
h:=\left(\begin{array}{ccc}
a & 0 & 0 \\
0 & B & C \\
0 & D & b
\end{array}\right) \in H
$$

with $a, b \in \mathbb{C}$, the conjugate

$$
\begin{aligned}
\sigma_{c} h \sigma_{c}^{-1} & =\left(\begin{array}{ccc}
\sqrt{c} & 0 & \sqrt{1-c} \\
0 & I_{n-2} & 0 \\
-\sqrt{1-c} & 0 & \sqrt{c}
\end{array}\right)\left(\begin{array}{lll}
a & 0 & 0 \\
0 & B & C \\
0 & D & b
\end{array}\right)\left(\begin{array}{ccc}
\sqrt{c} & 0 & -\sqrt{1-c} \\
0 & I_{n-2} & 0 \\
\sqrt{1-c} & 0 & \sqrt{c}
\end{array}\right) \\
& =\left(\begin{array}{ccc}
a \sqrt{c} & D \sqrt{1-c} & b \sqrt{1-c} \\
0 & B & C \\
-a \sqrt{1-c} & \sqrt{c} D & b \sqrt{c}
\end{array}\right)\left(\begin{array}{ccc}
\sqrt{c} & 0 & -\sqrt{1-c} \\
0 & I_{n-2} & 0 \\
\sqrt{1-c} & 0 & \sqrt{c}
\end{array}\right) \\
& =\left(\begin{array}{ccc}
a c+b-b c & D \sqrt{1-c} & (b-a) \sqrt{c} \sqrt{1-c} \\
C \sqrt{1-c} & B & C \sqrt{c} \\
(b-a) \sqrt{c} \sqrt{1-c} & D \sqrt{c} & a-a c+b c
\end{array}\right)
\end{aligned}
$$

is in $H$ if and only if $C=0, D=0$, and $b=a$, in which case

$$
\sigma_{c} h \sigma_{c}^{-1}=\left(\begin{array}{ccc}
a & 0 & 0 \\
0 & B & 0 \\
0 & 0 & a
\end{array}\right)=h
$$

with $B \in U(n-2)$ and $a^{2}=\operatorname{det}(B)^{-1}$. Thus, 


$$
\begin{aligned}
K_{\sigma_{c}}: & =H \cap H_{\sigma_{c}}=\left\{\left(\begin{array}{ccc}
a & 0 & 0 \\
0 & B & 0 \\
0 & 0 & a
\end{array}\right) \in S U(n): B \in U(n-2)\right\} \\
& =\left\{\left(\begin{array}{lll}
a & 0 & 0 \\
0 & B & 0 \\
0 & 0 & a
\end{array}\right): B \in U(n-2) \text { and } a^{2}=\operatorname{det}(B)^{-1}\right\}
\end{aligned}
$$

is a double covering $\tilde{U}(n-2)$ of $U(n-2)$ where $H_{\sigma_{c}}$ : $=\operatorname{Ad}_{\sigma_{c}} H=$ $\sigma_{c} H \sigma_{c}^{-1}$, and

$$
\mathfrak{k}_{\sigma_{c}}=\left\{\left(\begin{array}{ccc}
a & 0 & 0 \\
0 & B & 0 \\
0 & 0 & a
\end{array}\right): B \in \mathfrak{u}(n-2) \text { and }-2 a=\operatorname{tr}(B)\right\} \cong \mathfrak{u}(n-2) .
$$

It is not immediately clear that $H / K_{\sigma_{c}} \cong \mathbb{S}^{2 n-3}$ since $K_{\sigma_{c}} \cong \tilde{U}(n-2) \neq$ $U(n-2)$ and furthermore, under the following identification of $H$ and $U(n-1), K_{\sigma_{c}}$ is not identified with the standard canonically embedded $U(n-2)$, namely, $\left\{\left(\begin{array}{cc}1 & 0 \\ 0 & B\end{array}\right): B \in U(n-2)\right\}$.

Let us prove that $H / K_{\sigma_{c}} \cong \mathbb{S}^{2 n-3}$ and that $\pi$ on $H$ projects to the standard covariant Poisson structure on $\mathbb{S}^{2 n-3}$. Indeed, since $K_{\sigma_{c}}$ is a $\pi$ coisotropic subgroup of $H$ and the canonically embedded

$$
S U(n-1) \equiv H_{0}:=\left\{\left(\begin{array}{ll}
1 & 0 \\
0 & A
\end{array}\right): A \in S U(n-1)\right\}
$$

in $H$ is a Poisson-Lie subgroup of $H$, we have a Poisson embedding

$$
\iota: H_{0} /\left(H_{0} \cap K_{\sigma_{c}}\right) \rightarrow H / K_{\sigma_{c}}
$$

where the Poisson structures are projected from $\pi$. Note that $\iota$ is surjective (and hence is a diffeomorphism) since $H_{0} \cup K_{\sigma_{c}}$ generates the group $H$. Note also that

$$
H_{0} \cap K_{\sigma_{c}}=\left\{\left(\begin{array}{ccc}
1 & 0 & 0 \\
0 & B & 0 \\
0 & 0 & 1
\end{array}\right): B \in S U(n-2)\right\}
$$

the canonically embedded $S U(n-2)$ in $S U(n-1)$ and hence,

$$
H_{0} /\left(H_{0} \cap K_{\sigma_{c}}\right)=S U(n-1) / S U(n-2)=\mathbb{S}^{2 n-3} .
$$

This shows that, $H / K_{\sigma_{c}} \cong H_{0} /\left(H_{0} \cap K_{\sigma_{c}}\right)=\mathbb{S}^{2 n-3}$ the standard covariant Poisson sphere. Recall, that $H_{\sigma_{c}}=\operatorname{Ad}_{\sigma_{c}} H$ is $\pi$-coisotropic and $\pi$ projects to 
the non-standard covariant Poisson structure on $\mathbb{C} P^{n-1}=G / H_{\sigma_{c}}$. So with $H_{\sigma_{c}}$ being a Poisson-Lie subgroup of $(G, \pi)$, we have a Poisson embedding

$$
H / K_{\sigma_{c}}=\mathbb{S}^{2 n-3} \rightarrow G / H_{\sigma_{c}}=\mathbb{C} P^{n-1}
$$

of the standard Poisson $\mathbb{S}^{2 n-3}$ into the non-standard Poisson $\mathbb{C} P^{n-1}$.

Let now $k \neq 1$. We want to prove that

$$
\frac{K_{k}}{K_{k} \cap H_{\sigma_{c}}} \simeq \frac{\mathbb{S}^{2 k-1} \times \mathbb{S}^{2(n-k)-1}}{\mathbb{T}}
$$

as Poisson manifold, clarifying which is the Poisson structure on the right. Repeating the same argument as in the first part of the proof, we easily see that $K_{k} \cap H_{\sigma_{c}}$ consists of matrices

$$
\left(\begin{array}{cccc}
a & 0 & 0 & 0 \\
0 & B_{11} & 0 & 0 \\
0 & 0 & B_{22} & 0 \\
0 & 0 & 0 & a
\end{array}\right)
$$

where $B_{11} \in U(k-1), B_{22} \in U(n-k-1)$ and $a^{2}=\operatorname{det}\left(B_{11} B_{22}\right)^{-1}$. Now, since $K_{k} \cup J$ generates $U(k) \times U(n-k)$, we have

$$
\frac{K_{k}}{K_{k} \cap H_{\sigma_{c}}} \simeq \frac{U(k) \times U(n-k)}{J}
$$

where, as Poisson manifolds, $U(k) \times U(n-k)$ has the product Poisson structure (of standard Poisson $U(i)$ 's) and $J$ consists of matrices

$$
\left(\begin{array}{cccc}
a & 0 & 0 & 0 \\
0 & B_{11} & 0 & 0 \\
0 & 0 & B_{22} & 0 \\
0 & 0 & 0 & a
\end{array}\right)
$$

with $a \in U(1), B_{11} \in U(k-1), B_{22} \in U(n-k-1)$ (hence, $J$ is a Poisson-Lie subgroup of $U(k) \times U(n-k))$. We remark that

$$
\frac{U(k) \times U(n-k)}{1 \times U(k-1) \times U(n-k-1) \times 1} \simeq \mathbb{S}^{2 k-1} \times \mathbb{S}^{2(n-k)-1}
$$

with the product of standard Poisson structures on the right. It is just a quotient by a Poisson-Lie subgroup of $U(k) \times U(n-k)$. 
The canonical embedding

$$
1 \times U(k-1) \times U(n-k-1) \times 1 \subseteq J
$$

of Poisson-Lie groups induces a Poisson quotient map

$$
\frac{U(k) \times U(n-k)}{1 \times U(k-1) \times U(n-k-1) \times 1} \rightarrow \frac{U(k) \times U(n-k)}{J} .
$$

Since, the actions of the subgroups $1 \times U(k-1) \times U(n-k-1) \times 1$ and $\mathbb{T}=\left\{a \oplus I_{k-1} \oplus I_{n-k-1} \oplus a: a \in U(1)\right\}$ commute, $\mathbb{T}$ gives a well defined diagonal action on $\frac{U(k) \times U(n-k)}{1 \times U(k-1) \times U(n-k-1) \times 1} \simeq \mathbb{S}^{2 k-1} \times \mathbb{S}^{2(n-k)-1}$ such that the quotient map onto its orbit space coincides with the above quotient map.

The symplectic foliation of the standard covariant Poisson $\mathbb{S}^{2 k-1}$ consists of $\mathbb{T}$-families of $\mathbb{C}^{i}$ for $0 \leq i \leq k-1$ with the $\mathbb{T}$-action on $\mathbb{S}^{2 k-1}$ taking a leaf $\mathbb{C}^{i}$ to a leaf $\mathbb{C}^{i}$ in the same $\mathbb{T}$-family. So the symplectic foliation of $\mathbb{S}^{2 k-1} \times \mathbb{S}^{2(n-k)-1}$ consists of $\mathbb{T}^{2}$-families of $\mathbb{C}^{i} \times \mathbb{C}^{j}$ for $0 \leq j \leq n-k-1$ and hence the symplectic foliation of $\mathbb{S}^{2 k-1} \times \mathbb{S}^{2(n-k)-1} / \mathbb{T}$ consists of $\mathbb{T}$-families of $\mathbb{C}^{i} \times \mathbb{C}^{j}$ for $0 \leq i \leq k-1$ and $0 \leq j \leq n-k-1$.

\section{Remark 4.4.}

1. Note that $\operatorname{dim} X_{k}=\operatorname{dim} K_{k}-\operatorname{dim}\left(K_{k} \cap H_{\sigma}\right)=\left[k^{2}+(n-k)^{2}-1\right]-$ $\left[(k-1)^{2}+(n-k-1)^{2}\right]=2 n-3$ independently of $k$.

2. It is obvious that whenever $k \neq l, X_{k} \cap X_{l}$ is a union of lower dimensional symplectic leaves. Each such intersection is just the image under the Poisson embedding of the Poisson-Lie subgroup $K_{k} \cap K_{l}$.

3. The embedding $i_{\sigma}$ is the same as the Poisson map of Theorem 5 in [20]. To prove this statement consider that the map granted by proposition 4.1 can be constructed as follows: take $\left(v_{1}, \ldots, v_{n-1}\right)$ complex coordinates on the sphere, take $u^{\prime} \in U(n-1)$ with last column equal to $\left(v_{1}, \ldots, v_{n-1}\right)$ and consider $1 \oplus u^{\prime}$ as the matrix with first row and first column equal to $(1,0, \ldots, 0)$. Projecting this matrix with respect to $H_{\sigma}$ means projecting with $p \circ R_{\sigma}$ so that a direct computation shows that the Poisson map of proposition 4.1 is:

$$
\left(v_{1}, \ldots, v_{n-1}\right) \mapsto\left[\sqrt{1-c}, \sqrt{c} v_{1}, \ldots, \sqrt{c} v_{n-1}\right]
$$

(here [.] stands for equivalence class in $\mathbb{P}^{n-1}$ ) which is exactly the same map as in [20] (apart from composition with the obvious Poisson diffeomorphism $c \rightarrow 1-c$ ). It is remarkable that the connected 
components of the complementary of the union of the images of such embeddings are exactly the Poisson leaves of higher rank. Furthermore lower dimensional leaves can also be described as intersections of a suitable number of such images (the intersection of Poisson submanifolds being again a Poisson manifold), so that one can, in fact, completely describe the symplectic foliation of the complex projective space.

\subsection{Complex Grassmannians.}

In this section, we study the more general Grassmannian case. Let us fix once and for all the complete flag in $\mathbb{C}^{n}, V_{i}=\left\langle e_{n-i+1}, \ldots, e_{n}\right\rangle$ and let us give notations for the Schubert cell decomposition. Let $\left(a_{1}, \ldots, a_{k}\right)$ be a $k$-tuple of integers such that $0 \leq a_{1} \leq \ldots \leq a_{k} \leq n-k$, and denote with $\left[a_{1}, \ldots, a_{k}\right]$ the corresponding Schubert cell, i.e. the set of $k$-planes in $\mathbb{C}^{n}$ :

$$
\left[a_{1}, \ldots, a_{k}\right]=\left\{X \in G_{k}^{n} \mathbb{C} \mid \operatorname{dim}\left(X \cap V_{a_{i}+i}\right) \geq i\right\}
$$

Then, $\left[a_{1}, \ldots, a_{k}\right]$ is a cell of complex dimension $\sum_{i=1}^{k} a_{i}$. The relative position of cells is described by the so called Bruhat order:

$$
\left(a_{1}, \ldots, a_{k}\right) \leq\left(b_{1}, \ldots, b_{k}\right) \Longleftrightarrow a_{i} \leq b_{i} \quad \forall i=1, \ldots, k
$$

This is a partial ordering on the $k$-tuples of integers such that $\left(a_{1}, \ldots a_{k}\right) \leq$ $\left(b_{1}, \ldots, b_{k}\right)$ if and only if $\left[a_{1}, \ldots, a_{k}\right] \subseteq\left[b_{1}, \ldots, b_{k}\right]$. Notice that $\left[a_{1}, \ldots, a_{k-1}\right] \mapsto\left[0, a_{1}, \ldots, a_{k-1}\right]$ describes an embedding of $G_{k-1}^{n} \mathbb{C}$ into $G_{k}^{n} \mathbb{C}$

Now, we consider subgroups and their projections, starting with the standard case.

Theorem 4.5. For any $l=1, \ldots, n-1$, let $K_{l}=S(U(l) \times U(n-l))$ and let $G=S U(n)$. Then, we have:

1. There is a Poisson diffeomorphism

$$
\frac{K_{l}}{K_{l} \cap K_{k}}= \begin{cases}G_{k-l}^{n-l} \mathbb{C} & \text { if } l<k \\ \{e\} & \text { if } l=k \\ G_{k}^{l} \mathbb{C} & \text { otherwise }\end{cases}
$$


2. The image $X_{l}:=p\left(K_{l}\right)$ of $K_{l}$ under the projection $p: G \rightarrow G / K_{k}$ is the submanifold

$$
X_{l}=\left\{\begin{array}{ll}
{[\underbrace{0, \ldots, 0}_{l}, \underbrace{n-k, \ldots, n-k}_{k-l}]} & \text { if } l<k, \\
{[\underbrace{0, \ldots, 0]}_{k}]} & \text { if } l=k .
\end{array} .\right.
$$

Note that, we have the following inclusion relations: $X_{1} \supseteq \ldots \supseteq X_{k-1}$ and $X_{k+1} \subseteq \ldots \subseteq X_{n-1}$.

Proof. First, we note that

$$
K_{l} \cap K_{k}= \begin{cases}S(U(l) \times U(k-l) \times U(n-k)) & \text { if } l<k, \\ K_{k} & \text { if } l=k \\ S(U(k) \times U(l-k) \times U(n-l)) & \text { otherwise. }\end{cases}
$$

Furthermore, the union of the subgroups $1_{l} \times S U(n-l)$ and $K_{l} \cap K_{k}$ generates $K_{l}$ with

$$
\left(1_{l} \times S U(n-l)\right) \cap K_{l} \cap K_{k}=1_{l} \times S(U(k-l) \times U(n-k))
$$

if $l \leq k$, while the union of the subgroups $S U(l) \times 1_{n-l}$ and $K_{l} \cap K_{k}$ generates $K_{l}$ with

$$
\left(S U(l) \times 1_{n-l}\right) \cap K_{l} \cap K_{k}=S(U(k) \times U(l-k)) \times 1_{n-l}
$$

if $l>k$. So by lemma 4.1, we get Poisson diffeomorphisms

$$
\begin{aligned}
\frac{S U(n-l)}{S(U(k-l) \times U(n-k))} \rightarrow \frac{K_{l}}{K_{l} \cap K_{k}} ; & {[B] \mapsto\left(\begin{array}{cc}
I_{l} & 0 \\
0 & B
\end{array}\right) \quad l \leq k } \\
\frac{S U(l)}{S(U(k) \times U(l-k))} \rightarrow \frac{K_{l}}{K_{l} \cap K_{k}} ; & {[B] \mapsto\left(\begin{array}{cc}
B & 0 \\
0 & I_{n-l}
\end{array}\right) \quad l>k . }
\end{aligned}
$$

The rest of the theorem comes from direct computations.

We remark that different from the case of complex projective spaces, Poisson embeddings of lower dimensional homogeneous spaces do not cover 
the whole symplectic foliation for the complex Grassmannians which coincides with the Schubert cell decomposition.

Let us move to the non-standard situation. We are then considering

$$
G_{k}^{n} \mathbb{C} \simeq S U(n) / A d_{\sigma(c, k)} S(U(k) \times U(n-k))
$$

with the projected Poisson tensor $\tau_{\sigma_{c}}$. Let us consider the family of maximal Poisson-Lie subgroups $S(U(l) \times U(n-l)), 1 \leq l \leq n-1$. The problem is to describe, for every $l$, the image of $S(U(l) \times U(n-l))$ in $G_{k}^{n} \mathbb{C}$ and the Poisson manifold

$$
S(U(l) \times U(n-l)) /\left(S(U(l) \times U(n-l)) \cap A d_{\sigma(c, k)}(S(U(k) \times U(n-k))) .\right.
$$

Let $\mathbb{J}_{k}$ denote the $k \times k$ anti-diagonal matrix

$$
\mathbb{J}_{k}=\left(\begin{array}{cccc}
0 & \ldots & 0 & 1 \\
0 & \ldots & 1 & 0 \\
\vdots & \ddots & & \vdots \\
1 & 0 & \ldots & 0
\end{array}\right)=\sum_{i=1}^{k} e_{i, k-i+1}
$$

In the following, the subscript of $\mathbb{J}_{k}$ is often omitted since the size of $\mathbb{J}$ is varying and can be easily determined from its surrounding context. With this notation

$$
\sigma(c, k)=\left(\begin{array}{ccc}
\sqrt{c} \mathbb{I}_{k} & 0 & -\sqrt{1-c} \mathbb{J}_{k} \\
0 & \mathbb{I}_{n-2 k} & 0 \\
\sqrt{1-c} \mathbb{J}_{k} & 0 & \sqrt{c} \mathbb{I}_{k}
\end{array}\right) .
$$

Lemma 4.6. We have $K_{l} \cap A d_{\sigma(c, k)} K_{k}=K_{n-l} \cap A d_{\sigma(1-c, k)} K_{k}$.

Proof. First, of all $A d_{\sigma(c, k)} K_{k}$ consists of matrices of the form

$$
\begin{gathered}
\sigma(c, k)^{-1}\left(\begin{array}{ccc}
A & 0 & 0 \\
0 & B_{11} & B_{12} \\
0 & B_{21} & B_{22}
\end{array}\right) \sigma(c, k) \\
=\left(\begin{array}{ccc}
c A+(1-c) \mathbb{J} B_{22} \mathbb{J} & \sqrt{1-c} \mathbb{J} B_{21} & \sqrt{c(1-c)}\left(-A \mathbb{J}+\mathbb{J} B_{22}\right) \\
\sqrt{1-c} B_{12} \mathbb{J} & B_{11} & \sqrt{c} B_{12} \\
\sqrt{c(1-c)}\left(-\mathbb{J} A+B_{22} \mathbb{J}\right) & \sqrt{c} B_{21} & (1-c) \mathbb{J} A \mathbb{J}+c B_{22}
\end{array}\right)
\end{gathered}
$$


Now, the main point is to remark that $K_{n-i}=A d_{\mathbb{J}} K_{i}$ and that

$$
\begin{gathered}
\mathbb{J}\left(\begin{array}{ccc}
c A+(1-c) \mathbb{J} B_{22} \mathbb{J} & \sqrt{1-c} \mathbb{J} B_{21} & \sqrt{c(1-c)}\left(-A \mathbb{J}+\mathbb{J} B_{22}\right) \\
\sqrt{1-c} B_{12} \mathbb{J} & B_{11} & \sqrt{c} B_{12} \\
\sqrt{c(1-c)}\left(-\mathbb{J} A+B_{22} \mathbb{J}\right) & \sqrt{c} B_{21} & (1-c) \mathbb{J} A \mathbb{J}+c B_{22}
\end{array}\right) \mathbb{J} \\
=\left(\begin{array}{ccc}
(1-c) A+c \mathbb{J} B_{22} \mathbb{J} & \sqrt{c} \mathbb{J} B_{21} \mathbb{J} & \sqrt{c(1-c)}\left(-A \mathbb{J}+\mathbb{J} B_{22}\right) \\
\sqrt{c} \mathbb{J} B_{12} \mathbb{J} & \mathbb{J} B_{11} \mathbb{J} & \sqrt{1-c} \mathbb{J} B_{12} \\
\sqrt{c(1-c)}\left(-\mathbb{J} A+B_{22} \mathbb{J}\right) & \sqrt{1-c} B_{21} \mathbb{J} & c \mathbb{J} A \mathbb{J}+(1-c) B_{22}
\end{array}\right) \\
=A d_{\sigma(1-c, k)}\left(\begin{array}{ccc}
A & 0 & 0 \\
0 & \mathbb{J} B_{11} \mathbb{J} & \mathbb{J} B_{12} \\
0 & B_{21} \mathbb{J} & B_{22}
\end{array}\right)
\end{gathered}
$$

From this the claim follows.

Theorem 4.7. For any $l=1, \ldots, n-1$, let $K_{l}=S(U(l) \times U(n-l))$ and let $X_{l, k}=K_{l} /\left(K_{l} \cap A d_{\sigma(c, k)} K_{k}\right)$. Then, we have:

1. If $l<k$ or $l>n-k$, then $X_{l, k}$ is Poisson diffeomorphic to

$$
S U(n-l) /\left(S(U(|k-l|) \times U(|n-k-l|)) \times 1_{l}\right)
$$

with a non standard Poisson quotient structure. The image of $X_{l, k}$ is a Poisson submanifold of $G_{k}^{n} \mathbb{C}$ of codimension $l^{2}$ if $l<k$ and of codimension $(n-l)^{2}$ if $l>n-k$.

2. If $l=k$ or $l=n-k$, then $X_{l, k}$ is Poisson diffeomorphic to the Stiefel manifold

$$
V_{k}^{n-k} \mathbb{C}=U(n) /\left(1_{k} \times U(n-k)\right)
$$

with the standard quotient structure; its image in $G_{k}^{n} \mathbb{C}$ is therefore a Poisson submanifold of codimension $k^{2}$. Remark that when $2 k=n$, $V_{k}^{k} \mathbb{C} \simeq U(k)$ (with the standard Poisson structure).

3. If $k<l<n-k$, then $X_{l, k}$ is Poisson diffeomorphic to the quotient space

$$
\frac{V_{k}^{l} \mathbb{C} \times V_{k}^{n-l} \mathbb{C}}{U(k)}
$$

of the standard Poisson complex Stiefel manifold $V_{k}^{l} \mathbb{C} \times V_{k}^{n-l} \mathbb{C}$ by the diagonal action of $U(k)$. The image of the projection $X_{l, k}$ is a submanifold of $G_{k}^{n} \mathbb{C}$ of codimension $k^{2}$. 
Proof. Let us start with the case $l \leq k$. From the formula for $A d_{\sigma(c, k)} K_{k}$ described in the proof of the preceding lemma, we see that the subgroup $K_{l} \cap A d_{\sigma(c, k)} K_{k}$ consists of matrices of the form

$$
\left(\begin{array}{ccc}
A_{11} & 0 & 0 \\
0 & A^{\prime} & 0 \\
0 & 0 & \mathbb{J} A_{11} \mathbb{J}
\end{array}\right)
$$

where

$$
A^{\prime}=A d_{\sigma(c, k-l)}\left(\begin{array}{ccc}
A_{22} & 0 & 0 \\
0 & B_{11} & B_{12} \\
0 & B_{21} & B_{22}
\end{array}\right)
$$

such that the whole determinant is 1 , with blocks $A_{11} \in U(l), A_{22} \in U(k-l)$, $B_{11} \in M_{n-2 k}(\mathbb{C}), B_{22} \in U(k-l)$. When $l=k$ we get matrices

$$
\left(\begin{array}{ccc}
A_{11} & 0 & 0 \\
0 & B_{11} & 0 \\
0 & 0 & \mathbb{J} A_{11} \mathbb{J}
\end{array}\right)
$$

with $A_{11} \in U(k), B_{11} \in U(n-2 k)$ and $\operatorname{det} A_{11}^{2}=\operatorname{det} B^{-1}$. In this case, applying lemma 4.1 exactly as in the first part of the proof of Proposition 4.3 , we see that

$$
X_{l, k}=\frac{S U(n-l)}{\left\{\left(\begin{array}{cc}
A d_{\sigma(c, k-l)} A & 0 \\
0 & 1_{l}
\end{array}\right)\right\}}
$$

where $A \in S(U(k-l) \times U(n-k-l))$. Fix an auxiliary subgroup

$$
H_{0}=\left\{\left(\begin{array}{cc}
\mathbf{1}_{l} & 0 \\
0 & B
\end{array}\right): B \in S U(n-l)\right\}
$$

and notice that $H_{0} /\left(H_{0} \cap A d_{\sigma(c, k)} K_{k}\right)$ is Poisson diffeomorphic to $K_{l} /\left(K_{l} \cap\right.$ $\left.A d_{\sigma(c, k)} K_{k}\right)$. Next, $H_{0} /\left(H_{0} \cap A d_{\sigma(c, k)} K_{k}\right)$ is easily seen to be Poisson diffeomorphic to the standard Poisson quotients listed in the statement. In the special case, $l=k$ this yields the special case, of Stiefel manifolds. The symmetry provided by lemma 4.6 implies that the above results hold for $l \geq n-k$.

Now, we consider the remaining case. Take $k<l<n-k$ (and hence, $k \neq n / 2)$. Then, the intersection $K_{l} \cap A d_{\sigma(c, k)} K_{k}$ is given by:

$$
\left\{\left(\begin{array}{cccc}
A_{11} & 0 & 0 & 0 \\
0 & B_{11} & 0 & 0 \\
0 & 0 & B_{22} & 0 \\
0 & 0 & 0 & \mathbb{J}_{k} A_{11} \mathbb{J}_{k}
\end{array}\right)\right\}
$$


such that the whole determinant is one and $A_{11} \in U(k), B_{11} \in U(l-k)$, $B_{22} \in U(n-l-k)$. We have, then, considering that $K_{l} \cup J^{\prime}$ generates $U(l) \times U(n-l)$ and then applying lemma 8 .

$$
X_{l, k} \simeq \frac{U(l) \times U(n-l)}{J^{\prime}}
$$

where $U(l) \times U(n-l)$ has the product Poisson structure (of standard Poisson $U(i)$ 's) and $J^{\prime}$ consists of matrices

$$
\left(\begin{array}{cccc}
A_{11} & 0 & 0 & 0 \\
0 & B_{11} & 0 & 0 \\
0 & 0 & B_{22} & 0 \\
0 & 0 & 0 & \mathbb{J} A_{11 \mathbb{J}}
\end{array}\right)
$$

with no restrictions on determinants (hence $J^{\prime}$ is a Poisson-Lie subgroup of $U(l) \times U(n-l))$. We remark that

$$
\frac{U(l) \times U(n-l)}{1 \times U(l-k) \times U(n-k-l) \times 1} \simeq V_{k}^{l} \mathbb{C} \times V_{k}^{n-l} \mathbb{C}
$$

with the product of standard Poisson structures on Stiefel manifolds on the right. It is just a quotient by a Poisson-Lie subgroup of $U(l) \times U(n-l)$.

The canonical embedding

$$
1 \times U(l-k) \times U(n-k-l) \times 1 \subseteq J
$$

of Poisson-Lie groups induces a $U(l) \times U(n-l)$-equivariant, surjective Poisson map $V_{k}^{l} \mathbb{C} \times V_{k}^{n-l} \mathbb{C} \rightarrow X_{l, k}$, with fibre $U(k)$.

Since, the actions of the subgroups $1 \times U(l-k) \times U(n-k-l) \times 1$ and $U(k)=\left\{A \oplus I_{k-l} \oplus I_{n-k-l} \oplus A: A \in U(k)\right\}$ commute, $U(k)$ gives a well defined diagonal action on $V_{k}^{l} \mathbb{C} \times V_{k}^{n-l} \mathbb{C}$ such that the quotient map onto its orbit space coincides with the above quotient map.

The codimension statement is can be easily verified by computation.

\section{Remark 4.8.}

1. Explicit equations in Plücker coordinates for the embedded Poisson submanifolds $p_{\sigma_{c}}\left(K_{l}\right)$ can be obtained as in the $k=1$ case and are, at this point, matter of direct computations.

2. When $l<k$ or $l>n-k$ the Poisson homogeneous spaces $X_{k, l}$ are the non-standard version of the Poisson homogeneous spaces denoted by 
$U / K_{S}^{0}$ in [21], where $U=S U(n)$ and $S$, subset of the set of simple roots $\left\{\alpha_{1}, \ldots, \alpha_{n-l-1}\right\}$, in $\mathfrak{s u}(n)$, is given by deleting $\alpha_{k-l}$. Such Poisson manifold should be compared with the standard Poisson quotient $S U(n-l) / S(U(k-l) \times U(n-k-l)) \times 1_{l}$ in the sense of understanding whether the two belong to a Poisson pencil, as it is the case for projective and Grassmann manifolds.

3. As a last remark let us consider the maximal torus $T$ in $S U(n)$ then $\left.\pi\right|_{T}=0$. This implies that $T /\left(T \cap A d_{\sigma(c, k)} K_{k}\right)$ is a family of 0-dimensional symplectic leaves in Grassmannians which can be explicitly described:

$$
T \cap A d_{\sigma(c, k)} K_{k} \simeq \mathbb{T}^{n-k} \Rightarrow \frac{T}{T \cap A d_{\sigma(c, k)} K_{k}} \simeq \mathbb{T}^{k}
$$

where the image of such points in the Grassmannian is given by

$$
\left(t_{1}, \ldots, t_{k}\right) \mapsto\left\langle t_{j}\left(\sqrt{c} e_{n-j+1, n-j+1}-\sqrt{1-c} e_{j, n-j+1}\right) \mid j=1, \ldots, k\right\rangle .
$$

\section{References.}

[1] F. Bonechi and M. Zabzine, Poisson sigma models over group manifolds, Journ. Geom. Phys. 54, 173-196 (2005).

[2] M. Bordemann, G. Ginot, G. Halbout, H.-C. Herbig and S. Waldmann, Star-répresentation sur des sous-variétés coïsotropes, math.QA/0309321.

[3] A. Cattaneo and G. Felder, Coisotropic submanifolds in Poisson geometry, branes and Poisson $\sigma-$ models, Lett. Math. Phys. 69 (2004), 157175 .

[4] N. Ciccoli and F. Gavarini, Quantum duality principle for coisotropic subgroups and Poisson quotients, math.QA/0412465, Advances in Math 199 (2006) 104-135.

[5] P. Dazord and D. Sondaz, Groupes de Poisson affines, in 'Symplectic Geometry, Groupoids, and Integrable Systems', P. Dazord and A. Weinstein (Eds.), Springer-Verlag, 1991.

[6] M. S. Dijkhuizen and M. Noumi, A family of quantum projective spaces and related q-hypergeometric orthogonal polynomials, Trans. Amer. Math. Soc. 350 (1998), 3269-3296. 
[7] M.S. Dijkhuizen, M. Noumi and T. Sugitani, Multivariable AskeyWilson polynomials and quantum complex Grassmannians, in "Special functions, q-series and related topics", eds. M.E.H. Ismail et al., Fields. Inst. Comm. 14, AMS (1997), 167-177,

[8] V. G. Drinfeld, On Poisson homogeneous spaces of Poisson Lie groups, Theo. Math. Phys. 95 (1993), 226-227.

[9] S. Evens and J.-H. Lu, On the variety of Lagrangian subalgebras I, Ann. Sci. Ecole Norm. Sup. Paris. 34 (2001), 631-668.

[10] P. Foth and J.-H. Lu, A Poisson structure on compact symmetric spaces, Commun. Math. Phys. 251 (2004), 557-566.

[11] E. Karolinsky, The classification of Poisson homogeneous spaces of compact Poisson Lie groups, Mathematical Physics, Analysis, and Geometry. 3 (1996), 272-289.

[12] S. Khoroshkin, A. Radul, and V. Rubtsov, A family of Poisson structures on hermitian symmetric spaces, Comm. Math. Phys. 152 (1993), 299-315.

[13] J. H. Lu, Multiplicative and affine Poisson structures on Lie groups, Ph. D. thesis, Univ. of California, Berkeley. 1990.

[14] J.H. Lu, Poisson homogeneous spaces and Lie algebroids associated to Poisson actions, Duke Math. J. 86 (1997), 261-304.

[15] J. H. Lu and A. Weinstein, Poisson Lie groups, dressing transformations and Bruhat decompositions, J. Diff. Geom. 31 (1990), 501-526.

[16] J. H. Lu, Classification of $S U(2)$-covariant Poisson structures on $\mathbb{S}^{2}$, Comm. Math. Phys. 135 (1991), 229-231.

[17] G. B. Podkolzin and L. I. Vainerman, Quantum Stiefel manifolds and double cosets of quantum unitary group, Pac. J. Math. 138 (1999), 179199.

[18] A. J.-L. Sheu, Compact quantum groups and groupoid $C^{*}$-algebras, J. Func. Anal. 144 (1997), 371-393.

[19] A. J.-L. Sheu, Groupoid approach to quantum projective spaces, Contemp. Math. 228 (1998), 341-350. 
[20] A. J.-L. Sheu, Covariant Poisson structures on complex projective spaces, Comm. Anal. Geom. 10 (2002), 61-78.

[21] J. Stokman, The quantum orbit method for generalized flag manifolds, Math. Res. Lett. 10 (2003), 469-481.

[22] A. Weinstein, Affine Poisson structures, Intern. Journ. Math. 1 (1990), $343-360$. 